Discussion Paper \#2011-06

\title{
Distributions of Quadratic Functionals of the Fractional Brownian Motion Based on a Martingale Approximation
}

\author{
Katsuto Tanaka \\ Department of Economics, Hitotsubashi University \\ tanaka@stat.hit-u.ac.jp
}

June, 2011

\begin{abstract}
We discuss some computational problems associated with distributions of statistics arising from the fractional Brownian motion (fBm). In particular, we deal with (ratios of) its quadratic functionals. While it is easy in principle to deal with the standard Bm, the $\mathrm{fBm}$ is difficult to analyze because of its non-semimartingale nature. Here we suggest how to derive and compute the distributions of such functionals by using a martingale approximation. For this purpose we employ the Fredholm theory concerning the integral equations, illustrating how to compute the characteristic function via the Fredholm determinant. We also apply the present methodology to compute the fractional unit root distribution, and demonstrate some interesting moment properties.
\end{abstract}




\section{Introduction}

The present paper is concerned with distributions associated with the fractional Brownian motion $(\mathrm{fBm})$. The $\mathrm{fBm}$ is a continuous-time process that describes long-memory phenomena, and is now receiving much attention in the literature. It was invented by Kolmogorov (1940) and was largely developed by Mandelbrot and Van Ness (1968).

Let us denote by $\left\{B_{H}(t)\right\}$ the standard $\mathrm{fBm}$ defined on $[0,1]$, which is expressed as

$$
B_{H}(t)=c_{H}\left[\int_{-\infty}^{0}\left\{(t-u)^{H-1 / 2}-(-u)^{H-1 / 2}\right\} d W(u)+\int_{0}^{t}(t-u)^{H-1 / 2} d W(u)\right],
$$

where $c_{H}=(2 H \Gamma(3 / 2-H) /(\Gamma(H+1 / 2) \Gamma(2-2 H)))^{1 / 2}$ with $\Gamma(z)$ being the gamma function and $H$ the Hurst parameter assumed to be $1 / 2 \leq H<1$, whereas $\{W(t)\}$ is the standard $\mathrm{Bm}$. When $H=1 / 2$, the $\mathrm{fBm}$ reduces to the standard $\mathrm{Bm}$, that is, $B_{1 / 2}(t)=W(t)$. by

The process $\left\{B_{H}(t)\right\}$ is a zero-mean Gaussian process and its covariance kernel is given

$$
K_{H}(s, t)=\operatorname{Cov}\left(B_{H}(s), B_{H}(t)\right)=\frac{1}{2}\left(s^{2 H}+t^{2 H}-|s-t|^{2 H}\right) .
$$

It follows that the fBm has stationary increments in the sense that

$$
\mathrm{V}\left(B_{H}(t)-B_{H}(s)\right)=|s-t|^{2 H}, \quad(H \geq 1 / 2),
$$

and, as was shown in Gripenberg and Norros (1996), the following infinitesimal rule holds:

$$
\operatorname{Cov}\left(d B_{H}(s), d B_{H}(t)\right)=H(2 H-1)|s-t|^{2 H-2} d s d t, \quad(H>1 / 2) .
$$

It is seen that the increments of the $\mathrm{fBm}$ are not independent, but are positively correlated for $H>1 / 2$. Of course, when $H=1 / 2$, the increments are independent and it holds that

$$
\operatorname{Cov}\left(d B_{1 / 2}(s), d B_{1 / 2}(t)\right)=\operatorname{Cov}(d W(s), d W(t))=\delta_{s t} d t
$$

where $\delta_{s t}$ is Kronecker's delta.

The fBm may be regarded as a continuous version of the discrete-time unit root process whose innovation error follows a long-memory process. More specifically, let us consider

$$
y_{j}=y_{j-1}+v_{j}, \quad(1-L)^{H-1 / 2} v_{j}=\varepsilon_{j}, \quad y_{0}=0, \quad(j=1, \cdots, T) .
$$

where $L$ is the lag-operator, $\left\{\varepsilon_{j}\right\} \sim$ i.i.d. $(0,1)$, whereas $\left\{v_{j}\right\}$ is a stationary long-memory process generated by

$$
v_{j}=(1-L)^{-(H-1 / 2)} \varepsilon_{j}=\sum_{k=0}^{\infty} \frac{\Gamma(k+H-1 / 2)}{\Gamma(H-1 / 2) \Gamma(k+1)} \varepsilon_{j-k} .
$$

Then it holds (Davydov (1970)) that

$$
\frac{c_{H} \Gamma(H+1 / 2)}{T^{H}} y_{[T t]} \Rightarrow B_{H}(t)
$$

where $\Rightarrow$ signifies weak convergence as $T \rightarrow \infty$.

It is sometimes the case that the infinite sum in (6) is truncated so that we obtain

$$
v_{j}^{*}=(1-L)^{-(H-1 / 2)}\left\{I(j>0) \varepsilon_{j}\right\}=\sum_{k=0}^{j-1} \frac{\Gamma(k+H-1 / 2)}{\Gamma(H-1 / 2) \Gamma(k+1)} \varepsilon_{j-k},
$$


where $I(j>0)$ takes 1 for $j>0$ and 0 otherwise. In this case we have

$$
y_{j}^{*}=y_{j-1}^{*}+v_{j}^{*}, \quad(1-L)^{H-1 / 2} v_{j}^{*}=I(j>0) \varepsilon_{j}, \quad y_{0}^{*}=0, \quad(j=1, \cdots, T),
$$

and the following weak convergence holds (Marinucci and Robinson 1999):

$$
\frac{c_{H} \Gamma(H+1 / 2)}{T^{H}} y_{[T t]}^{*} \Rightarrow B_{H}^{*}(t)=c_{H} \int_{0}^{t}(t-u)^{H-1 / 2} d W(u) .
$$

It is seen that $B_{H}^{*}(t)$ is a portion of $B_{H}(t)$ in (1). Since the omitted term $B_{H}(t)-B_{H}^{*}(t)$ is independent of $B_{H}^{*}(t)$, it holds that $\mathrm{V}\left(B_{H}(t)\right)>\mathrm{V}\left(B_{H}^{*}(t)\right)$. In fact we have

$$
\mathrm{V}\left(B_{H}(t)\right)=t^{2 H}>\mathrm{V}\left(B_{H}^{*}(t)\right)=\frac{c_{H}^{2}}{2 H} t^{2 H}=\frac{\Gamma(3 / 2-H)}{\Gamma(H+1 / 2) \Gamma(2-2 H)} t^{2 H} .
$$

The truncated process $\left\{B_{H}^{*}(t)\right\}$ is called the type II $\mathrm{fBm}$, whereas the standard $\mathrm{fBm}$ $\left\{B_{H}(t)\right\}$ the type I fBm (Marinucci and Robinson 1999).

An advantage of the use of $B_{H}^{*}(t)$ is that it can accommodate any positive value of $H$ unlike $B_{H}(t)$. There are some other differences between the two processes, as was investigated in Davidson and Hashimzade (2009). In this paper, however, we stick to $B_{H}(t)$ mainly because its covariance kernel is simply expressed as in (2), while that of $B_{H}^{*}(t)$ cannot be explicitly given. In fact, we have

$$
\operatorname{Cov}\left(B_{H}^{*}(s), B_{H}^{*}(t)\right)=c_{H}^{2} \int_{0}^{\min (s, t)}((s-u)(t-u))^{H-1 / 2} d u,
$$

which complicates the subsequent discussion.

Returning to $\left\{B_{H}(t)\right\}$, the most important difference between $W(t)$ and $B_{H}(t)$ is that the latter is neither a martingale nor a semimartingale so that we cannot use the stochastic Ito calculus based on semimartingales. The relation (4) is a direct consequence of this.

One of the main purposes of the present paper is to derive the distribution of

$$
S_{H}=\int_{0}^{1} B_{H}^{2}(t) d t \stackrel{\mathcal{D}}{=} \int_{0}^{1} \int_{0}^{1} K_{H}(s, t) d W(s) d W(t),
$$

where $\stackrel{\mathcal{D}}{=}$ denotes the equality in the sense of distribution, and its equivalence will be shown in the next section together with another equivalent expression. The quantity $S_{H}$ plays an important role in the estimation and testing problems associated with the fBm. It holds that, when $H=1 / 2$,

$$
S_{H}=\int_{0}^{1} W^{2}(t) d t \stackrel{\mathcal{D}}{=} \int_{0}^{1} \int_{0}^{1}[1-\max (s, t)] d W(s) d W(t), \quad(H=1 / 2),
$$

and its distribution has been well studied. The distribution of $S_{H}$ for $H \neq 1 / 2$, however, is still unknown.

In Section 2 we describe some basic properties associated with quadratic functionals of the standard Bm. Section 3 discusses how to derive the characteristic function (c.f.) of such functionals, where we present two approaches for this purpose. The first approach is based on Girsanov's theorem concerning the transformation of measures induced by the fBm. The second approach uses the theory of integral equations of Fredholm type. Both approaches are useful for dealing with quadratic functionals of the Bm, but it turns out that neither approach is successful when we deal with the fBm. In Section 4 we 
consider approximating the distribution using a martingale approximation to the $\mathrm{fBm}$. For this purpose the second approach proves to be useful, which enables us to compute the approximate densitiy of $S_{H}$. We also present graphs of those densities for various values of $H$. Section 5 applies our methodology to compute the approximate fractional unit root distribution, where an interesting moment property is found and a conjecture that the same moment property holds is given for the true fractional unit root distribution. Concluding remarks are given in Section 6, and the proofs of theorems are provided in the Appendix.

\section{Quadratic functionals of the Bm}

Let us consider the following statistic

$$
S=\int_{0}^{1} \int_{0}^{1} K(s, t) d W(s) d W(t)
$$

where the kernel $K(s, t)$ is assumed to be continuous, symmetric and positive definite. To define positive definiteness of the kernel $K(s, t)$, we consider the integral equation for $\lambda$ and $f(t)$

$$
f(t)=\lambda \int_{0}^{1} K(s, t) f(s) d s .
$$

A value $\lambda$ for which this integral equation possesses a nonvanishing continuous solution is called an eigenvalue of $K(s, t)$; the corresponding solution $f(t)$ is called an eigenfunction for the eigenvalue $\lambda$. The maximum number of linearly independent solutions $f(t)$ corresponding to $\lambda$ is called the multiplicity of $\lambda$. Then $K(s, t)$ is positive definite if all of the eigenvalues are positive. Note that zero is never an eigenvalue. We also note that, since $K(s, t)$ is continuous and symmetric, the sequence of eigenfunctions $\left\{f_{n}(t)\right\}$ can be taken to be orthonormal, which we assume in subsequent discussions.

The statistic $S$ in (11) naturally arises from the usual qudratic form. To see this let us consider the following quadratic form:

$$
S_{T}=\frac{1}{T} \varepsilon^{\prime} A \varepsilon=\frac{1}{T} \sum_{j=1}^{T} \sum_{k=1}^{T} A_{T}(j, k) \varepsilon_{j} \varepsilon_{k},
$$

where $\varepsilon=\left(\varepsilon_{1}, \ldots \varepsilon_{T}\right)^{\prime}$ with $\left\{\varepsilon_{j}\right\} \sim$ i.i.d. $(0,1)$, whereas $A$ is a $T \times T$ symmetric matrix with $A_{T}(j, k)$ in the $(j, k)$ th element. Then, if $A_{T}(j, k)$ converges uniformly to $K(s, t)$ in the sense that

$$
\lim _{T \rightarrow \infty} \max _{1 \leq j, k \leq T}\left|A_{T}(j, k)-K(j / T, k / T)\right|=0,
$$

it holds that $S_{T} \Rightarrow S$ (Nabeya and Tanaka (1988)).

The statistic $S$ defined by the double integral as in (11) has two equivalent expressions in the sense of distribution. First of all, Mercer's theorem (Hochstadt (1973)) gives us the expansion

$$
K(s, t)=\sum_{n=1}^{\infty} \frac{1}{\lambda_{n}} f_{n}(s) f_{n}(t)
$$

where $\left\{\lambda_{n}\right\}$ is a sequence of eigenvalues repeated as many times as their multiplisities, whereas $\left\{f_{n}(t)\right\}$ is an orthonormal sequence of eigenfunctions corresponding to $\lambda_{n}$. It is 
ensured that the series on the right side converges absolutely and uniformly to $K(s, t)$. Then we have

$$
\int_{0}^{1} \int_{0}^{1} K(s, t) d W(s) d W(t)=\sum_{n=1}^{\infty} \frac{1}{\lambda_{n}}\left(\int_{0}^{1} f_{n}(t) d W(t)\right)^{2} \stackrel{\mathcal{D}}{=} \sum_{n=1}^{\infty} \frac{1}{\lambda_{n}} Z_{n}^{2}
$$

where $\left\{Z_{n}\right\} \sim \operatorname{NID}(0,1)$ and $\lambda_{n}$ is repeated as many times as its multiplicity.

On the other hand, the Karhunen-Loève expansion (Loève (1978)) enables us to define

$$
X(t)=\sum_{n=1}^{\infty} \frac{f_{n}(t)}{\sqrt{\lambda_{n}}} Z_{n}
$$

where the series converges uniformly on $[0,1]$ in the mean square sense, and $\lambda_{n}$ is repeated as many times as its multiplicity. Then it holds that

$$
\int_{0}^{1} X^{2}(t) d t=\int_{0}^{1} \sum_{m=1}^{\infty} \sum_{n=1}^{\infty} \frac{f_{m}(t) f_{n}(t)}{\sqrt{\lambda_{m}} \sqrt{\lambda_{n}}} Z_{m} Z_{n} d t=\sum_{n=1}^{\infty} \frac{1}{\lambda_{n}} Z_{n}^{2} .
$$

It follows from (14) and (16) that

$$
S=\int_{0}^{1} \int_{0}^{1} K(s, t) d W(s) d W(t) \stackrel{\mathcal{D}}{=} \int_{0}^{1} X^{2}(t) d t \stackrel{\mathcal{D}}{=} \sum_{n=1}^{\infty} \frac{1}{\lambda_{n}} Z_{n}^{2},
$$

Note also that, given $\{X(t)\}$, it always holds that

$$
\int_{0}^{1} X^{2}(t) d t \stackrel{\mathcal{D}}{=} \int_{0}^{1} \int_{0}^{1} \operatorname{Cov}(X(s), X(t)) d W(s) d W(t)
$$

on which the distributional relationship presented in (10) is based.

In terms of the derivation of the characteristic function (c.f.) of $S$, the expression on the extreme right side of (17) is the simplest of the three. In fact we readily have

$$
\phi(\theta)=\mathrm{E}\left(e^{i \theta S}\right)=\prod_{n=1}^{\infty}\left(1-\frac{2 i \theta}{\lambda_{n}}\right)^{-1 / 2} .
$$

This, however, is possible only if the sequence $\left\{\lambda_{n}\right\}$ is known, which is rarely the case. Thus we need to take a different approach based on the other expressions in (17), which we discuss in the next section.

\section{Derivation of the c.f.}

In this section we present two methods for deriving the c.f. of the statistic $S$ defined in (17). Section 3.1 deals with the simple integral expression, and then we treat the double integral expression in Section 3.2.

\subsection{Stochastic process approach via Girsanov's theorem}

Let $(C, \mathcal{B}(C))$ be the measurable space of continuous functions on $[0,1]$ with the associated Borel $\sigma$-field generated under the supremum norm. Then consider the statistic

$$
S=\int_{0}^{1} X^{2}(t) d t
$$


where $\{X(t)\}$ is the Gaussian stochastic process that can be expanded as in (15). In particular, we consider, as $\mathrm{X}(\mathrm{t})$,

$$
d Y_{H}^{\alpha}(t)=-\alpha Y_{H}^{\alpha}(t) d t+d B_{H}(t), \quad Y_{H}^{\alpha}(0)=0,
$$

where $\alpha$ is a fixed parameter. The process $\left\{Y_{H}^{\alpha}(t)\right\}$ is called the fractional OrnsteinUhlenbeck (O-U) process (see, for details, Kleptsyna and Le Breton (2002)), which reduces to the standard $\mathrm{O}-\mathrm{U}$ process when $H=1 / 2$. The process $\left\{Y_{H}^{\alpha}(t)\right\}$ can arise from the following discrete-time process:

$$
y_{j}=\rho y_{j-1}+v_{j}, \quad(1-L)^{H-1 / 2} v_{j}=\varepsilon_{j}, \quad y_{0}=0, \quad(j=1, \cdots, T),
$$

where $\rho=1-\alpha / T$ and $\left\{\varepsilon_{j}\right\} \sim$ i.i.d. $(0,1)$. Then it holds that

$$
\frac{c_{H} \Gamma(H+1 / 2)}{T^{H}} y_{[T t]} \Rightarrow Y_{H}^{\alpha}(t) .
$$

We also define the auxiliary process

$$
d Y_{H}^{\beta}(t)=-\beta Y_{H}^{\beta}(t) d t+d B_{H}(t), \quad Y_{H}^{\beta}(0)=0,
$$

where $\beta$ is a parameter to be determined later.

Let $\mu_{Y_{H}^{\alpha}}$ and $\mu_{Y_{H}^{\beta}}$ be the probability measures on $(C, \mathcal{B}(C))$ induced by $\left\{Y_{H}^{\alpha}(t)\right\}$ and $\left\{Y_{H}^{\beta}(t)\right\}$, respectively, by the relation

$$
\mu_{Y_{H}^{\alpha}}(A)=P\left(\omega: Y_{H}^{\alpha} \in A\right), \quad \mu_{Y_{H}^{\beta}}(A)=P\left(\omega: Y_{H}^{\beta} \in A\right), \quad A \in \mathcal{B}(C) .
$$

Then the fractional version of Girsanov's theorem was obtained by Kleptsyna, Le Breton and Roubaud (2000), which says that measures $\mu_{Y_{H}^{\alpha}}$ and $\mu_{Y_{H}^{\beta}}$ are equivalent and the Radon-Nikodym derivative is given by

$$
\frac{d \mu_{Y_{H}^{\alpha}}}{d \mu_{Y_{H}^{\beta}}}\left(Y_{H}^{\beta}\right)=\exp \left[(\beta-\alpha) \int_{0}^{1} Q_{H}^{\beta}(t) d Z_{H}^{\beta}(t)-\frac{\alpha^{2}-\beta^{2}}{2} \int_{0}^{1}\left\{Q_{H}^{\beta}(t)\right\}^{2} d v_{H}(t)\right],
$$

where $v_{H}(t)=t^{2-2 H} \Gamma(3 / 2-H) /(2 H \Gamma(3-2 H) \Gamma(H+1 / 2))$, and

$$
\begin{aligned}
Q_{H}^{\beta}(t) & =\frac{d}{d v_{H}(t)} \int_{0}^{t} l_{H}(t, s) Y_{H}^{\beta}(s) d s, \\
Z_{H}^{\beta}(t) & =\int_{0}^{t} l_{H}(t, s) d Y_{H}^{\beta}(s), \\
M_{H}(t) & =\int_{0}^{t} l_{H}(t, s) d B_{H}(s),
\end{aligned}
$$

with $l_{H}(t, s)=(2 H \Gamma(3 / 2-H) \Gamma(H+1 / 2))^{-1}(s(t-s))^{1 / 2-H}$.

It is shown in Norros, Valkeila and Virtamo (1999) that the process $\left\{M_{H}(t)\right\}$ in $(25)$ is a Gaussian martingale with the variance $v_{H}(t)$, which has the inverse relationship

$$
B_{H}(t)=\int_{0}^{t} L_{H}(t, s) d M_{H}(s), \quad L_{H}(t, s)=H(2 H-1) \int_{s}^{t} u^{H-1 / 2}(u-s)^{H-3 / 2} d u,
$$

so that $\left\{M_{H}(t)\right\}$ generates the same filtration as $\left\{B_{H}(t)\right\}$ and is called the fundamental martingale. The process $\left\{M_{H}(t)\right\}$ will play an important role in this paper. 
The process $\left\{Q_{H}^{\beta}(t)\right\}$ in (23) admits the representation

$$
Q_{H}^{\beta}(t)=\frac{\eta_{H}}{4(1-H)}\left\{t^{2 H-1} Z_{H}^{\beta}(t)+\int_{0}^{t} s^{2 H-1} d Z_{H}^{\beta}(s)\right\}, \eta_{H}=\frac{2 H \Gamma(3-2 H) \Gamma(H+1 / 2)}{\gamma(3 / 2-H)},
$$

and will be important in constructing the likelihood function, as is discussed shortly.

The process $\left\{Z_{H}^{\beta}(t)\right\}$ in (24) admits the representation

$$
Z_{H}^{\beta}(t)=-\beta \int_{0}^{t} Q_{H}^{\beta}(s) d v_{H}(s)+M_{H}(t)
$$

and is seen to be a Gaussian semimartingale. In particular, it reduces to the martingale $M_{H}(t)$ when $\beta=0$. It also holds that

$$
Y_{H}^{\beta}(t)=\int_{0}^{t} L_{H}(t, s) d Z_{H}^{\beta}(s),
$$

so that the natural filtration generated by the semimartingale $\left\{Z_{H}^{\beta}(t)\right\}$ and the fractional O-U process $\left\{Y_{H}^{\beta}(t)\right\}$ coincides.

The Radon-Nikodym derivative in (22) is composed of various complicated processes. Suppose that $H=1 / 2$. Then it is seen that we have $M_{1 / 2}(t)=B_{1 / 2}(t)=W(t), \eta_{1 / 2}=1$, $v_{1 / 2}(t)=t$, and $Q_{1 / 2}^{\beta}(t)=Z_{1 / 2}^{\beta}(t)=Y_{1 / 2}^{\beta}(t)$ so that both $Q_{1 / 2}^{\beta}(t)$ and $Z_{1 / 2}^{\beta}(t)$ follow the same standard O-U process as $Y_{1 / 2}^{\beta}(t)$. Thus the Radon-Nikodym derivative reduces to

$$
\frac{d \mu_{Y_{1 / 2}^{\alpha}}}{d \mu_{Y_{1 / 2}^{\beta}}}\left(Y_{1 / 2}^{\beta}\right)=\exp \left[(\beta-\alpha) \int_{0}^{1} Y_{1 / 2}^{\beta}(t) d Y_{1 / 2}^{\beta}(t)-\frac{\alpha^{2}-\beta^{2}}{2} \int_{0}^{1}\left\{Y_{1 / 2}^{\beta}(t)\right\}^{2} d t\right] \text {. }
$$

This formula was initially given by Liptser and Shiryaev (1977), and is useful for deriving the moment generating function (m.g.f.) of the integral of the square of the standard $\mathrm{O}-\mathrm{U}$ process $\left\{Y_{1 / 2}^{\alpha}(t)\right\}$ in the following way:

$$
\begin{aligned}
m(\theta) & =\mathrm{E}\left[\exp \left\{\theta \int_{0}^{1}\left(Y_{1 / 2}^{\alpha}(t)\right)^{2} d t\right\}\right]=\mathrm{E}\left[\exp \left\{\theta \int_{0}^{1}\left(Y_{1 / 2}^{\beta}(t)\right)^{2} d t\right\} \frac{d \mu_{Y_{1 / 2}^{\alpha}}}{d \mu_{Y_{1 / 2}^{\beta}}}\left(Y_{1 / 2}^{\beta}\right)\right] \\
& =\mathrm{E}\left[\exp \left\{\left(\theta-\frac{\alpha^{2}-\beta^{2}}{2}\right) \int_{0}^{1}\left(Y_{1 / 2}^{\beta}(t)\right)^{2} d t+(\beta-\alpha) \int_{0}^{1} Y_{1 / 2}^{\beta}(t) d Y_{1 / 2}^{\beta}(t)\right\}\right] \\
& =\mathrm{E}\left[\exp \left\{\frac{\beta-\alpha}{2}\left(\left(Y_{1 / 2}^{\beta}(1)\right)^{2}-1\right)\right\}\right]=e^{\alpha / 2}\left[\cosh \beta+\alpha \frac{\sinh \beta}{\beta}\right]^{-1 / 2},
\end{aligned}
$$

where we have chosen $\beta=\sqrt{\alpha^{2}-2 \theta}$ so that the term containing the integral of $\left(Y_{1 / 2}^{\beta}(t)\right)^{2}$ vanishes. We have also used the Ito calculus $d\left(Y_{1 / 2}^{\beta}(t)\right)^{2}=2 Y_{1 / 2}^{\beta}(t) d Y_{1 / 2}^{\beta}(t)+d t$ and the fact that $Y_{1 / 2}^{\beta}(1)$ is normally distributed with mean 0 and variance $\left(1-e^{-2 \beta}\right) /(2 \beta)$. In particular, putting $\alpha=0$, we obtain

$$
\mathrm{E}\left[\exp \left\{\theta \int_{0}^{1} W^{2}(t) d t\right\}\right]=(\cosh \sqrt{-2 \theta})^{-1 / 2}=(\cos \sqrt{2 \theta})^{-1 / 2} .
$$


The above argument, however, cannot be carried over directly to the fractional O$\mathrm{U}$ process. In fact, what we can compute via the fractional Girsanov theorem is the following:

$$
\begin{aligned}
m(\theta) & =\mathrm{E}\left[\exp \left\{\theta \int_{0}^{1}\left(Q_{H}^{\alpha}(t)\right)^{2} d v_{H}(t)\right\}\right] \\
& =\mathrm{E}\left[\exp \left\{\theta \int_{0}^{1}\left(Q_{H}^{\beta}(t)\right)^{2} d v_{H}(t)\right\} \frac{d \mu_{Y_{H}^{\alpha}}}{d \mu_{Y_{H}^{\beta}}}\left(Y_{H}^{\beta}\right)\right] \\
& =\mathrm{E}\left[\exp \left\{\left(\theta-\frac{\alpha^{2}-\beta^{2}}{2}\right) \int_{0}^{1}\left(Q_{H}^{\beta}(t)\right)^{2} d v_{H}(t)+(\beta-\alpha) \int_{0}^{1} Q_{H}^{\beta}(t) d Z_{H}^{\beta}(t)\right\}\right] \\
& =\mathrm{E}\left[\exp \left\{(\beta-\alpha) \int_{0}^{1} Q_{H}^{\beta}(t) d Z_{H}^{\beta}(t)\right\}\right],
\end{aligned}
$$

where we have chosen $\beta=\sqrt{\alpha^{2}-2 \theta}$ so that the term containing the integral of $\left(Q_{H}^{\beta}(t)\right)^{2}$ vanishes. Even when $\alpha=0$, the deterministic process $v_{H}(t)$ is independent of $\alpha$, and

$$
Q_{H}^{\alpha}(t)=\frac{d}{d v_{H}(t)} \int_{0}^{t} l_{H}(t, s) B_{H}(s) d s, \quad(\alpha=0) .
$$

Thus it is seen that the fractional Girsanov theorem is not directly related with the fBm $B_{H}(t)$, but with its linear functional $Q_{H}^{\alpha}(t)$.

For general $H(\neq 1 / 2)$ and nonzero $\alpha$, Kleptsyna and Le Breton (2002) went on to compute (26). This computation is much involved, but is useful when we discuss the MLE of the parameter $\alpha$. In fact, on the basis of the Radon-Nikodym derivative, the likelihood function $L(\alpha)$ for the fractional O-U process in (19) is obtained as

$$
L(\alpha)=\exp \left[-\alpha \int_{0}^{1} Q_{H}^{\alpha}(t) d Z_{H}^{\alpha}(t)-\frac{\alpha^{2}}{2} \int_{0}^{1}\left\{Q_{H}^{\alpha}(t)\right\}^{2} d v_{H}(t)\right] .
$$

Then the MLE $\tilde{\alpha}$ of $\alpha$ is given by

$$
\tilde{\alpha}=-\int_{0}^{1} Q_{H}^{\alpha}(t) d Z_{H}^{\alpha}(t) / \int_{0}^{1}\left\{Q_{H}^{\alpha}(t)\right\}^{2} d v_{H}(t),
$$

which will also be regarded as the weak limit of $-T(\tilde{\rho}-1)$ as $T \rightarrow \infty$, where $\tilde{\rho}$ is the MLE of $\rho$ in the discrete-time model (20) under the assumption $\left\{\varepsilon_{j}\right\} \sim$ N.I.D.(0,1). In particular, when $H=1 / 2,-\tilde{\alpha}$ corresponds to the near-unit root distribution, that is,

$$
-\tilde{\alpha}=\int_{0}^{1} Y_{1 / 2}^{\alpha}(t) d Y_{1 / 2}^{\alpha}(t) / \int_{0}^{1}\left(Y_{1 / 2}^{\alpha}(t)\right)^{2} d t, \quad(H=1 / 2)
$$

Of course, when $H=1 / 2$ and $\alpha=0,-\tilde{\alpha}$ reduces to the unit root distribution

$$
-\tilde{\alpha}=\int_{0}^{1} W(t) d W(t) / \int_{0}^{1} W^{2}(t) d t, \quad(H=1 / 2, \alpha=0) .
$$

To derive the distribution of the MLE of $\alpha$ for the general case, we need to compute

$$
\begin{aligned}
\psi\left(\theta_{1}, \theta_{2}\right) & =\mathrm{E}\left[\exp \left\{\theta_{1} \int_{0}^{1} Q_{H}^{\alpha}(t) d Z_{H}^{\alpha}(t)+\theta_{2} \int_{0}^{1}\left\{Q_{H}^{\alpha}(t)\right\}^{2} d v_{H}(t)\right\}\right] \\
& =\mathrm{E}\left[\exp \left\{\left(\theta_{1}+\beta-\alpha\right) \int_{0}^{1} Q_{H}^{\beta}(t) d Z_{H}^{\beta}(t)\right\}\right],
\end{aligned}
$$


where the parameter $\beta$ is now chosen as $\beta=\sqrt{\alpha^{2}-2 \theta_{2}}$. It is seen that the computation involved in (27) is essentially the same as in (26). Note that the argument here has assumed that the Hurst parameter $H$ is known. If it is unknown, the problem becomes highly complicated and needs another considereation. We do not pursue the matter here. In any case, the above argument does not give us the c.f. of our statistic $S_{H}$ in (10).

In conclusion, the standard Girsanov theorem is useful for deriving the distribution of quadratic functionals of the standard O-U process, whereas the fractional Girsanov theorem computes the c.f. of quadratic functional of the quantity quite different from the $\mathrm{fBm}$. It seems that the stochastic process approach via Girsanov's theorem is of little help in the present situation.

\subsection{Fredholm approach via the Fredholm determinant}

Here we deal with the statistic of the form

$$
S=\int_{0}^{1} \int_{0}^{1} K(s, t) d W(s) d W(t)
$$

where $K(s, t)$ is assumed to be continuous, symmetric and nearly definite. By nearly definiteness we mean that all but a finite number of eigenvalues of $K(s, t)$ have the same sign. This weakened assumption may be necessary when we deal with a ratio statistic of the following form:

$$
R=\int_{0}^{1} K_{N}(s, t) d W(s) d W(t) / \int_{0}^{1} K_{D}(s, t) d W(s) d W(t),
$$

where $K_{D}(s, t)$ is positive definite. Then we deal with

$$
P(R<x)=P\left(\int_{0}^{1} \int_{0}^{1}\left[x K_{D}(s, t)-K_{N}(s, t)\right] d W(s) d W(t)>0\right) .
$$

Here the kernel $x K_{D}(s, t)-K_{N}(s, t)$ is not ensured to be definite, although $K_{D}(s, t)$ is assumed to be definite. Thus the assumption of $K(s, t)$ being nearly definite is necessary. Note that Mercer's theorem is still valid so that the expansion described in (13) and the distributional equivalence in (14) continue to hold.

Under the above assumptions it was shown by Anderson and Darling (1952) (see also Hochstadt (1973)) that

$$
\phi(\theta)=\mathrm{E}\left(e^{i \theta S}\right)=(D(2 i \theta))^{-1 / 2},
$$

where $D(\lambda)$ is the Fredholm determinant (FD) of the kernel $K(s, t)$.

The FD of the kernel $K(s, t)$ is defined as

$$
\begin{aligned}
D(\lambda) & =\lim _{T \rightarrow \infty} D_{T}(\lambda)=\prod_{n=1}^{\infty}\left(1-\frac{\lambda}{\lambda_{n}}\right)^{l_{n}} \\
& =\sum_{n=0}^{\infty} \frac{(-\lambda)^{n}}{n !} \int_{0}^{1} \cdots \int_{0}^{1}\left|\left(\begin{array}{ccc}
K\left(t_{1}, t_{1}\right) & \cdots & K\left(t_{n}, t_{1}\right) \\
\vdots & & \vdots \\
K\left(t_{n}, t_{1}\right) & \cdots & K\left(t_{n}, t_{n}\right)
\end{array}\right)\right| d t_{1} \cdots d t_{n},
\end{aligned}
$$

where $\lambda_{1}<\lambda_{2}<\cdots$ are eigenvalues of $K$ and $l_{n}$ is the multiplicity of $\lambda_{n}$, whereas

$$
D_{T}(\lambda)=\left|I_{T}-\frac{\lambda}{T} K_{T}\right|, \quad K_{T}=[(K(j / T, k / T))]: T \times T .
$$


It is seen that $D(\lambda)$ is the limit of the determinant whose zero yields eigenvalues in the matrix theory. The series in (29) is shown to converge for all $\lambda$, that is, $D(\lambda)$ is an integral function with $D(0)=1$. It also holds that every zero of $D(\lambda)$ is an eigenvalue of $K$, and in turn every eigenvalue of $K$ is a zero of $D(\lambda)$. Thus the FD contains necessary and sufficient information about eigenvalues. It, however, is usually impossible to compute the FD following (29).

An alternative method for obtaining the FD is demonstrated in Nabeya and Tanaka $(1988,1990)$ and Tanaka (1996), where a set of sufficient conditions for a function of $\lambda$ to be the FD is described as follows:

Theorem 1. Let $K(s, t)$ be continuous, symmetric, and nearly definite with a sequence of eigenvalues $\left\{\lambda_{n}\right\}$. Suppose that $\tilde{D}(\lambda)$ is an integral function of $\lambda$ with $\tilde{D}(0)=1$. Then $\tilde{D}(\lambda)$ becomes the FD of $K$ if

i) every zero of $\tilde{D}(\lambda)$ is an eigenvalue of $K$, and in turn every eigenvalue of $K$ is a zero of $\tilde{D}(\lambda)$;

ii) $\tilde{D}(\lambda)$ can be expanded as

$$
\tilde{D}(\lambda)=\prod_{n=1}^{\infty}\left(1-\frac{\lambda}{\lambda_{n}}\right)^{l_{n}}
$$

where $l_{n}$ is equal to the multiplicity of $\lambda_{n}$.

A word may be in order. If $\tilde{D}(\lambda)$ satisfies the conditions described in Theorem 1 , $\tilde{D}^{2}(\lambda)$, for example, is not the FD. This is because the zero of $\tilde{D}^{2}(\lambda)$ at $\lambda_{n}$ is of order $2 l_{n}$, whereas the multiplicity of $\lambda_{n}$ is $l_{n}$.

To obtain a candidate $\tilde{D}(\lambda)$ for the FD of $K$, we work with a differential equation with some boundary conditions equivalent to the integral equation (12). As an illustration, let us consider

$$
K_{1}(s, t)=\min (s, t)-\frac{1}{2}(s+t)+\frac{1}{2}(s-t)^{2}+\frac{1}{12},
$$

which was used in Watson (1961) for a goodness-of-fit test on a circle, and is positive definite so that every eigenvalue is positive. Differentiating on both sides of (12) with respect to $t$ twice replacing $K$ by $K_{1}$ in (31), we have

$$
f^{\prime}(t)=\lambda\left[\int_{t}^{1} f(s) d s+\int_{0}^{1}\left(t-s-\frac{1}{2}\right) f(s) d s\right], f^{\prime \prime}(t)+\lambda f(t)=\lambda \int_{0}^{1} f(s) d s .
$$

Then it can be shown that the integral equation (12) with $K$ replaced by $K_{1}$ is equivalent to the equation $f(t)=c_{1} \cos \sqrt{\lambda} t+c_{2} \sin \sqrt{\lambda} t+c_{3}$ with three boundary conditions:

$$
f(0)=f(1), \quad f^{\prime}(0)=f^{\prime}(1), \quad f(0)=\lambda\left[\frac{c_{3}}{12}+\frac{1}{2} \int_{0}^{1}\left(t^{2}-t\right) f(t) d t\right],
$$

where $c_{1}, c_{2}$ and $c_{3}$ are arbitrary constants. From these boundary conditions we have the homogeneous equation $M_{1}(\lambda) \boldsymbol{c}=\mathbf{0}$, where $\boldsymbol{c}=\left(c_{1}, c_{2}, c_{3}\right)^{\prime}$ and

$$
M_{1}(\lambda)=\left(\begin{array}{ccc}
1-\cos \sqrt{\lambda} & -\sin \sqrt{\lambda} & 0 \\
\sin \sqrt{\lambda} & 1-\cos \sqrt{\lambda} & 0 \\
\cos \sqrt{\lambda}-\frac{2 \sin \sqrt{\lambda}}{\sqrt{\lambda}}-1 & \sin \sqrt{\lambda}+\frac{2 \cos \sqrt{\lambda}-2}{\sqrt{\lambda}} & -2
\end{array}\right) .
$$


The solution $f(t)$ must be nonvanishing, which occurs only when $\boldsymbol{c} \neq \mathbf{0}$. Then the equation $M_{1}(\lambda) \boldsymbol{c}=\mathbf{0}$ implies that

$$
\left|M_{1}(\lambda)\right|=-4(1-\cos \sqrt{\lambda})=-8\left(\sin \frac{\sqrt{\lambda}}{2}\right)^{2}=0 .
$$

Thus $\lambda(\neq 0)$ is an eigenvalue if and only if $\sin (\sqrt{\lambda} / 2)=0$, which yields $\sqrt{\lambda_{n}}=2 n \pi(n=$ $1,2, \ldots)$. To determine the multiplicity we have the following theorem (Tanaka (1996)), which describes nothing but the dimension of a null space in the theory of matrices.

Theorem 2. Suppose that the integral equation (12) is equivalent to a differential equation with some boundary conditions. Suppose further that the latter is equivalent to

$$
f(t)=c_{1} \phi_{1}(t)+\cdots+c_{r} \phi_{r}(t), \quad M(\lambda) \boldsymbol{c}=\mathbf{0},
$$

where $\phi_{1}(t), \cdots, \phi_{r}(t)$ are linearly independent, continuous functions, whereas $M(\lambda)$ is the $r \times r$ coefficient matrix of the system of linear homogeneous equations in $\boldsymbol{c}=\left(c_{1}, \cdots, c_{r}\right)^{\prime}$. Then the multiplicity $l_{n}$ of the eigenvalue $\lambda_{n}$ is given by

$$
l_{n}=r-\operatorname{rank}\left(M\left(\lambda_{n}\right)\right) .
$$

Applying Theorem 2 to the matrix $M_{1}(\lambda)$ in (33) with $\sqrt{\lambda_{n}}=2 n \pi$, it is seen that $\operatorname{rank}\left(M_{1}\left(\lambda_{n}\right)\right)=1$ for each $n$ so that the multiplicity of $\lambda_{n}$ is 2 for each $n$. Therefore, allowing for the infinite product expansion for $\sin x$, we obtain, as a candidate of the FD,

$$
\tilde{D}_{1}(\lambda)=\left(\sin \frac{\sqrt{\lambda}}{2} / \frac{\sqrt{\lambda}}{2}\right)^{2}=\prod_{n=1}^{\infty}\left(1-\frac{\lambda}{(2 n \pi)^{2}}\right)^{2} .
$$

It can now be checked that $\tilde{D}_{1}(\lambda)$ satisfies the two conditions in Theorem 1 . Thus the FD of $K_{1}(s, t)$ in (31) is given by (34) so that we have, from (28),

$$
\mathrm{E}\left[\exp \left\{i \theta \int_{0}^{1} \int_{0}^{1} K_{1}(s, t) d W(s) d W(t)\right\}\right]=(\tilde{D}(2 i \theta))^{-1 / 2}=\left(\sin \sqrt{\frac{i \theta}{2}} / \sqrt{\frac{i \theta}{2}}\right)^{-1} .
$$

The fact that the multiplicity of each eigenvalue is 2 can also be seen by considering the following equality derived from (13):

$$
\int_{0}^{1} K_{1}(t, t) d t=\int_{0}^{1} \frac{1}{12} d t=\frac{1}{12}=\sum_{n=1}^{\infty} \frac{1}{\lambda_{n}}
$$

where $\lambda_{n}=(2 n \pi)^{2}$. Since $\sum_{n=1}^{\infty} 1 /(2 n \pi)^{2}=1 / 24$, each eigenvalue must be repeated twice in the above sum.

We also note in passing that it holds that

$$
S_{1}=\int_{0}^{1} \int_{0}^{1} K_{1}(s, t) d W(s) d W(t) \stackrel{\mathcal{D}}{=} \int_{0}^{1}\left(W_{B}(t)-\int_{0}^{1} W_{B}(u) d u\right)^{2} d t,
$$

where $\left\{W_{B}(t)\right\}=\{W(t)-t W(1)\}$ is the standard Brownian bridge process, and the distributional equivalence above comes from the fact that

$$
\operatorname{Cov}\left(W_{B}(s)-\int_{0}^{1} W_{B}(u) d u, W_{B}(t)-\int_{0}^{1} W_{B}(u) d u\right)=K_{1}(s, t) .
$$


Thus the c.f. of $S_{1}$ may also be obtained by the stochastic process approach discussed in the previous subsection after some algebra.

We now turn to the quadratic functional of the $\mathrm{fBm}$ and try to derive the FD of $K_{H}(s, t)$ defined in (2). It, however, turns out that differentiation on the both sides of the integral equation (12) with the kernel $K_{H}$ yields no plausible differential equation we can work with. In fact, putting $\alpha=H-1 / 2$, we have

$$
\begin{aligned}
& f^{\prime}(t)=\frac{\lambda(2 \alpha+1)}{2}\left[t^{2 \alpha} \int_{0}^{1} f(s) d s-\int_{0}^{t}(t-s)^{2 \alpha} f(s) d s+\int_{t}^{1}(s-t)^{2 \alpha} f(s) d s\right], \\
& f^{\prime \prime}(t)=\lambda \alpha(2 \alpha+1)\left[t^{2 \alpha-1} \int_{0}^{1} f(s) d s-\int_{0}^{t}(t-s)^{2 \alpha-1} f(s) d s-\int_{t}^{1}(s-t)^{2 \alpha-1} f(s) d s\right] .
\end{aligned}
$$

Because of the existence of the term $|s-t|^{2 \alpha+1}=|s-t|^{2 H}$ in the kernel $K_{H}(s, t)$, it is impossible to obtain any plausible differential equation.

Thus the Fredholm approach also seems inapplicable to the kernel $K_{H}(s, t)$. Nonetheless we can still compute the FD of a kernel arising from a martingale approximation to the $\mathrm{fBm}$, which we shall discuss in the next section.

\section{Case of the fBm}

In this section we approximate the nonsemimartingale $\mathrm{fBm}\left\{B_{H}(t)\right\}$ by a martingale process. The statistic $S_{H}$ in (10) is replaced accordingly so that the Fredholm approach discussed in the last section is amenable to deriving the FD.

\subsection{Martingale approximation to the $\mathrm{fBm}$}

As was mentioned in Section 2, Norros, Valkeila and Virtamo (1999) showed that the stochastic process $\left\{M_{H}(t)\right\}$ defined in (25), that is,

$$
M_{H}(t)=\kappa_{H}^{-1} \int_{0}^{t}(s(t-s))^{1 / 2-H} d B_{H}(s), \quad \kappa_{H}=2 H \Gamma(3 / 2-H) \Gamma(H+1 / 2),
$$

is a Gaussian martingale, and reduces to $B_{1 / 2}(t)=W(t)$ when $H=1 / 2$. Some basic properties of $\left\{M_{H}(t)\right\}$ follow.

(a) $\left\{M_{H}(t)\right\}$ is a Gaussian martingale and thus has independent increments.

(b) Increments of $M_{H}(t)$ are independent of $B_{H}(s)$ for $t>s$.

(c) $\operatorname{Cov}\left(M_{H}(s), M_{H}(t)\right)=a_{H}^{2}(\min (s, t))^{2-2 H}, \quad a_{H}=\sqrt{\frac{\Gamma(3 / 2-H)}{2 H \Gamma(H+1 / 2) \Gamma(3-2 H)}}$,

(d) $\operatorname{Corr}\left(B_{H}(t), M_{H}(t)\right)=a_{H}^{-1}$

(e) $M_{H}(t) \stackrel{\mathcal{D}}{=} b_{H} \int_{0}^{t} s^{1 / 2-H} d W(s), \quad b_{H}=\sqrt{2(1-H)} a_{H}$.

The property (b) is a consequence of the martingale property of $\left\{M_{H}(t)\right\}$ and (35). The covariance structure in (c) also leads to the martingale nature of $\left\{M_{H}(t)\right\}$. The correlation property $(\mathrm{d})$ is of our most concern in terms of approximating $B_{H}(t)$ by $M_{H}(t)$. It is noticed that the correlation depends on $H$, but is the same for all $t$. The property (e) is useful for expressing the quadratic functional of $M_{H}(t)$ in terms of $W(t)$. 
Figure 1 is concerned with the property (d) mentioned above and draws the correlation between $B_{H}(t)$ and $M_{H}(t)$ as a function of $H(0<H<1)$. It is seen that the correlation is very close to 1 for $H>0.4$. In fact it is greater than 0.99 for $H>0.4$, which is, of course, equal to 1 when $H=1 / 2$.

\section{Figure 1}

For $H>1 / 2$, we are now led to approximate $B_{H}(t)$ by $C_{H}(t)$, where

$$
C_{H}(t)=a_{H}^{-1} t^{2 H-1} M_{H}(t) \stackrel{\mathcal{D}}{=} \sqrt{2(1-H)} t^{2 H-1} \int_{0}^{t} s^{-(H-1 / 2)} d W(s) .
$$

Note that the above approximation ensures that $\operatorname{Corr}\left(B_{H}(t), C_{H}(t)\right)=a_{H}^{-1}$ and

$$
\mathrm{E}\left(B_{H}(t)\right)=\mathrm{E}\left(C_{H}(t)\right)=0, \quad \mathrm{~V}\left(B_{H}(t)\right)=\mathrm{V}\left(C_{H}(t)\right)=t^{2 H} .
$$

Note, however, the covariance structure of $\left\{C_{H}(t)\right\}$ is different from that of $\left\{B_{H}(t)\right\}$ since

$$
\operatorname{Cov}\left(C_{H}(s), C_{H}(t)\right)=(s t)^{2 H-1}(\min (s, t))^{2-2 H} .
$$

We now consider, as an approximation to $S_{H}$ in $(10)$,

$$
\begin{aligned}
\tilde{S}_{H} & =\int_{0}^{1} C_{H}^{2}(t) d t=a_{H}^{-2} \int_{0}^{1}\left(t^{2 H-1} M_{H}(t)\right)^{2} d t \\
& \stackrel{\mathcal{D}}{=} \frac{1-2 \alpha}{4 \alpha+1} \int_{0}^{1} \int_{0}^{1}\left[1-(\max (s, t))^{4 \alpha+1}\right] s^{-\alpha} t^{-\alpha} d W(s) d W(t) \\
& \stackrel{\mathcal{D}}{=} \int_{0}^{1} \int_{0}^{1}(s t)^{2 \alpha}(\min (s, t))^{1-2 \alpha} d W(s) d W(t),
\end{aligned}
$$

where we have put $\alpha=H-1 / 2(0 \leq \alpha<1 / 2)$. Here the equality in (39) comes from expressing $M_{H}(t)$ by using the property (e) mentioned above, whereas (40) from the expression for $\operatorname{Cov}\left(C_{H}(s), C_{H}(t)\right)$ described in (38). Note that, when $H=1 / 2(\alpha=0)$, both expressions coincide in the sense of distribution with $S_{1 / 2}$. It is also of interest to note that, when $H=1(\alpha=1 / 2)$, the expression in (40) is equal to $S_{H}=S_{1}$, whereas (39) is meaningless, although we exclude that case. To see the differences of the true and approximate kernels, Figure 2 draws $D_{H}(s, t)=\operatorname{Cov}\left(C_{H}(s), C_{H}(t)\right)-\operatorname{Cov}\left(B_{H}(s), B_{H}(t)\right)$ for $H=0.3$, whereas Figure 3 draws $D_{H}(s, t)$ for $H=0.8$. It is seen that the difference is quite large when $H=0.3$ in comparison with the case of $H=0.8$. This reflects the correlation sturucture between $B_{H}(t)$ and $C_{H}(t)$ shown in Figure 1.

\section{Figure $2 \quad$ Figure 3}

We can now compute the FD of the kernel in (39) or (40), which we discuss in the next subsection.

\subsection{Approximate distribution}


We use the Fredholm approach to compute the distribution of $\tilde{S}_{H}$ given in (39) or (40). For this purpose we obtain the following theorem, which is proved in the Appendix.

Theorem 3. For the random variable $\tilde{S}_{H}$ given in (39) or (40) it holds that

$$
\phi_{H}(\theta)=\mathrm{E}\left(e^{i \theta \tilde{S}_{H}}\right)=\left[\left(\frac{\delta}{2}\right)^{\nu} \Gamma(1-\nu) J_{-\nu}(\delta)\right]^{-1 / 2},
$$

where

$$
\delta=\frac{\sqrt{1-2 \alpha}}{\alpha+1} \sqrt{2 i \theta}, \quad \nu=\frac{2 \alpha+1 / 2}{\alpha+1}, \quad \alpha=H-1 / 2,
$$

and $J_{\nu}(z)$ is the Bessel function of the first kind defined by

$$
J_{\nu}(z)=\sum_{k=0}^{\infty} \frac{(-1)^{k}(z / 2)^{2 k+\nu}}{k ! \Gamma(k+\nu+1)}
$$

Note that, when $H=1 / 2(\alpha=0)$, we have $\delta=\sqrt{2 i \theta}, \nu=1 / 2$ and $J_{-1 / 2}(z)=$ $\sqrt{2 /(\pi z)} \cos z$ so that

$$
\phi_{H}(\theta)=(\cos \sqrt{2 i \theta})^{-1 / 2}, \quad(H=1 / 2),
$$

which is the c.f. of $\int_{0}^{1} W^{2}(t) d t$.

On the basis of Theorem 3, we can compute numerically the distribution function of the positive random variable $\tilde{S}_{H}$ using the inversion formula:

$$
P\left(\tilde{S}_{H}<x\right)=\frac{1}{\pi} \int_{0}^{\infty} \operatorname{Re}\left[\frac{1-e^{-i \theta x}}{i \theta} \phi_{H}(\theta)\right] d \theta .
$$

The probability density of $\tilde{S}_{H}$ can be computed by $d P\left(\tilde{S}_{H}<x\right) / d x$ or numerical differentiation of the distribution function and the computation of integration can be done by Simpson's rule. Care, however, needs to be taken in the computation of the c.f. because it contains the square root of complex-valued quantities. To overcome this difficulty a modified algorithm as shown in Tanaka (1996) may be necessary.

Figure 4 presents graphs of probability densities of $\tilde{S}_{H}$ for various values of $H$. The density of $\tilde{S}_{1 / 2}$ is exact, but the others are approximations to the density of $S_{H}$. Note that both $S_{H}$ and $\tilde{S}_{H}$ converge to $\chi^{2}(1) / 3$ as $H \rightarrow 1$. The limiting density as $H \rightarrow 1$ becomes monotone and diverges at 0 , unlike the densities for $H<1$.

\section{Figure 4}

To see the closeness of the densities of $\tilde{S}_{H}$ to $S_{H}$, we have compared the first two moments of $S_{H}$ and $\tilde{S}_{H}$. It holds that

$$
\mathrm{E}\left(S_{H}\right)=\mathrm{E}\left(\tilde{S}_{H}\right)=\int_{0}^{1} t^{2 H} d t=\frac{1}{2 H+1},
$$




$$
\begin{aligned}
& \mathrm{V}\left(S_{H}\right)=2 \int_{0}^{1} \int_{0}^{1}\left\{\frac{1}{2}\left(s^{2 H}+t^{2 H}-|s-t|^{2 H}\right)\right\}^{2} d s d t \\
& \mathrm{~V}\left(\tilde{S}_{H}\right)=\left.\frac{d^{2} \phi_{H}(-i \theta)}{d \theta^{2}}\right|_{\theta=0}-\mathrm{E}^{2}\left(\tilde{S}_{H}\right)=\frac{4 H+5}{3(2 H+1)^{2}}-\frac{1}{(2 H+1)^{2}}=\frac{2}{3(2 H+1)} .
\end{aligned}
$$

Table 1 reports $\mathrm{V}\left(S_{H}\right)$ and $\mathrm{V}\left(\tilde{S}_{H}\right)$ for various values of $H$, where the case for $H=1$ was also presented as a limiting case. It is seen that the variances of the approximate densities coincide with the true ones up to the second decimal places as a whole. There is no evidence that the approximation is poor, but more details remain to be investigated. Table 2 reports percent points of $\tilde{S}_{H}$ for the same values of $H$ as in Table 1 . It is anticipated that the density behaves quite differently as $H$ approaches 1 .

Table 1

Table 2

As was noted before, the approximation may be worse when $H<0.4$ because of low correlation between $S_{H}$ and $\tilde{S}_{H}$. We, however, are still able to compute the densities of $\tilde{S}_{H}$. Figure 5 draws densities of $\tilde{S}_{H}$ for $H=0.2,0.3, \cdots, 0.9$. It is seen that these densities are unimodal and are shifted to the right with larger variances as $H$ becomes smaller.

\section{Figure 5}

\section{Fractional unit root distribution}

In this section we apply the martingale approximation discussed in the previous section to compute the density of the fractional unit root distribution. Let us consider

$$
y_{j}=\rho y_{j-1}+v_{j},(1-L)^{H-1 / 2} v_{j}=u_{j}, \text { true } \rho=1, y_{0}=0,(j=1, \cdots, T),
$$

where $1 / 2<H<1$ and $\left\{u_{j}\right\}$ is a short-memory stationary process like the ARMA process with the long-run variance $\sigma^{2}$. Then the error process $\left\{v_{j}\right\}$ is a long-memory process that admits the following weak convergence:

$$
\frac{c_{H} \Gamma(H+1 / 2)}{\sigma T^{H}} y_{[T t]}=\frac{c_{H} \Gamma(H+1 / 2)}{\sigma T^{H}} \sum_{j=1}^{[T t]} v_{j} \Rightarrow B_{H}(t),
$$

where $c_{H}$ is a constant defined in (1). Then, as was shown in Sowell (1990), the OLSE $\hat{\rho}$ of $\rho$ follows

$$
T(\hat{\rho}-1) \Rightarrow R_{H}=\frac{\frac{1}{2} B_{H}^{2}(1)}{\int_{0}^{1} B_{H}^{2}(t) d t}, \quad(1 / 2<H<1) .
$$

It is noticed that $R_{H}$ is a functional of $\left\{B_{H}(t)\right\}$ dealt with in previous sections. We now try to approximate the distribution of $R_{H}$ by using the martingale approximation.

\subsection{The FD associated with the approximate distribution}


Let us consider the following approximation:

$$
R_{H}=\frac{\frac{1}{2} B_{H}^{2}(1)}{\int_{0}^{1} B_{H}^{2}(t) d t} \approx \tilde{R}_{H}=\frac{\frac{1}{2} M_{H}^{2}(1)}{\int_{0}^{1}\left(t^{2 H-1} M_{H}(t)\right)^{2} d t} .
$$

Then we deal with the following approximate distribution:

$$
\begin{aligned}
P\left(\tilde{R}_{H}<x\right) & =P\left(x \int_{0}^{1}\left(t^{2 H-1} M_{H}(t)\right)^{2} d t-\frac{1}{2} M_{H}^{2}(1)>0\right) \\
& =P\left(\int_{0}^{1} \int_{0}^{1} K_{H}(s, t ; x) d W(s) d W(t)>0\right)
\end{aligned}
$$

where, putting $\alpha=H-1 / 2(0<\alpha<1 / 2)$,

$$
K_{H}(s, t ; x)=\frac{x}{4 \alpha+1}\left(1-(\max (s, t))^{4 \alpha+1}\right) s^{-\alpha} t^{-\alpha}-\frac{1}{2} s^{-\alpha} t^{-\alpha} .
$$

Now we have the following theorem concerning the FD of the kernel $K_{H}(s, t ; x)$ :

Theorem 4. The FD of $K_{H}(s, t ; x)$ in (45) is given by

$$
D_{H}(\lambda ; x)=\left(\frac{\kappa}{2}\right)^{\nu} \Gamma(1-\nu)\left(J_{-\nu}(\kappa)+\frac{\lambda}{2} \frac{J_{1-\nu}(\kappa)}{\sqrt{\lambda x}}\right)
$$

where

$$
\kappa=\frac{\sqrt{\lambda x}}{H+1 / 2}, \quad \nu=\frac{2 H-1 / 2}{H+1 / 2} .
$$

When $H=1 / 2(\alpha=0)$, we have $\kappa=\sqrt{\lambda x}, \nu=1 / 2$, and noting that $J_{-1 / 2}(z)=$ $\sqrt{2 /(\pi z)} \cos z$ and $J_{1 / 2}(z)=\sqrt{2 /(\pi z)} \sin z$, it is seen that the FD of $K_{1 / 2}(s, t ; x)=x(1-$ $\max (s, t))-1 / 2$ associated with $\tilde{R}_{1 / 2}$ is given by

$$
D_{1 / 2}(\lambda ; x)=\cos \sqrt{\lambda x}+\frac{\lambda}{2} \frac{\sin \sqrt{\lambda x}}{\sqrt{\lambda x}},
$$

which is also the FD associated with $R_{1 / 2}$. On the basis of Theorem 4 we can compute the approximate distribution of $R_{H}$ employing Imhof's formula:

$$
P\left(\tilde{R}_{H}<x\right)=\frac{1}{2}+\frac{1}{\pi} \int_{0}^{\infty} \frac{1}{\theta} \operatorname{Im}\left[\left(D_{H}(2 i \theta ; x)\right)^{-1 / 2}\right] d \theta .
$$

Figure 6 draws probability densities of $\tilde{R}_{H}$ for various values of $H(\geq 1 / 2)$. These are approximate densities of the original fractional unit root distribution $R_{H}$, although the density with $H=1 / 2$ is exact. The densities have a positive support and diverge at the origin, and are shifted to the right as $H$ becomes large. These densities have an interesting moment property, which we discuss in the next subsection.

\section{Figure 6}




\subsection{An interesting moment property}

Here we first compute the moments of the approximate unit root distributions. For this purpose let us define the joint m.g.f. of the numerator $\tilde{U}_{H}$ and the denominator $\tilde{V}_{H}$ of $\tilde{R}_{H}$ in (44) by

$$
\begin{aligned}
m_{H}\left(\theta_{1}, \theta_{2}\right) & =\mathrm{E}\left[\exp \left\{\theta_{1} \tilde{U}_{H}+\theta_{2} \tilde{V}_{H}\right\}\right] \\
& =\mathrm{E}\left[\exp \left\{\theta_{1} \frac{1}{2} M_{H}^{2}(1)+\theta_{2} \int_{0}^{1}\left(t^{2 H-1} M_{H}(t)\right)^{2} d t\right\}\right] \\
& =\left[D_{H}\left(-2 \theta_{1} ;-\theta_{2} / \theta_{1}\right)\right]^{-1 / 2} \\
& =\left[\left(\frac{\sqrt{2 \theta_{2}}}{2(\alpha+1)}\right)^{\nu} \Gamma(1-\nu)\left(J_{-\nu}\left(\frac{\sqrt{2 \theta_{2}}}{\alpha+1}\right)-\frac{\theta_{1}}{\sqrt{2 \theta_{2}}} J_{1-\nu}\left(\frac{\sqrt{2 \theta_{2}}}{\alpha+1}\right)\right)\right]^{-1 / 2},
\end{aligned}
$$

where $\alpha=H-1 / 2, \nu=(2 \alpha+1 / 2) /(\alpha+1)$. Note that, when $H=1 / 2$, we have

$$
m_{H}\left(\theta_{1}, \theta_{2}\right)=\left[\cos \sqrt{2 \theta_{2}}-\theta_{1} \frac{\sin \sqrt{2 \theta_{2}}}{\sqrt{2 \theta_{2}}}\right]^{-1 / 2}, \quad(H=1 / 2) .
$$

The $j$ th order moment of $\tilde{R}_{H}$ can then be computed as

$$
\mathrm{E}\left(\tilde{R}_{H}^{j}\right)=\mathrm{E}\left[\left(\frac{M_{H}^{2}(1) / 2}{\int_{0}^{1}\left(t^{2 H-1} M_{H}(t)\right)^{2} d t}\right)^{j}\right]=\left.\frac{1}{(j-1) !} \int_{0}^{\infty} \theta_{2}^{j-1} \frac{\partial^{j} m\left(\theta_{1},-\theta_{2}\right)}{\partial \theta_{1}^{j}}\right|_{\theta_{1}=0} d \theta_{2} .
$$

In particular, when $j=1$, we have the following result.

Theorem 5. The mean of $\tilde{R}_{H}$ is given by

$$
\mathrm{E}\left(\tilde{R}_{H}\right)=\mathrm{E}\left(\frac{\tilde{U}_{H}}{\tilde{V}_{H}}\right)=\mathrm{E}\left(\frac{M_{H}^{2}(1) / 2}{\int_{0}^{1}\left(t^{2 H-1} M_{H}(t)\right)^{2} d t}\right)=\alpha+1=H+\frac{1}{2} .
$$

Noting that $\mathrm{E}\left(M_{H}^{2}(t)\right)=a_{H}^{2} t^{2-2 H}$, the simple expression as was obtained in Theorem 5 leads us to conclude that

$$
\mathrm{E}\left(\tilde{R}_{H}\right)=\mathrm{E}\left(\frac{\tilde{U}_{H}}{\tilde{V}_{H}}\right)=\frac{\mathrm{E}\left(\tilde{U}_{H}\right)}{\mathrm{E}\left(\tilde{V}_{H}\right)}=\frac{1}{2} / \frac{1}{2 H+1}=H+\frac{1}{2} .
$$

This implies that the expectation of the ratio is equal to the ratio of expectations, which occurs if $\tilde{R}_{H}$ is independent of $\tilde{V}_{H}$. This, however, is not the case, which can be checked by verifying that

$$
\mathrm{E}\left(\tilde{R}_{H}^{2} \tilde{V}_{H}^{2}\right)=\mathrm{E}\left(\tilde{U}_{H}^{2}\right) \neq \mathrm{E}\left(\tilde{R}_{H}^{2}\right) \mathrm{E}\left(\tilde{V}_{H}^{2}\right)
$$

In fact, when $H=1 / 2$, we have

$$
\begin{aligned}
\mathrm{E}\left(\tilde{R}_{1 / 2}^{2}\right) & =\mathrm{E}\left[\left(\frac{1}{2} W^{2}(1) / \int_{0}^{1} W^{2}(t) d t\right)^{2}\right] \\
& =\left.\int_{0}^{\infty} \theta_{2} \frac{\partial^{2}}{\partial \theta_{2}^{2}}\left[\cosh \sqrt{2 \theta_{2}}-\theta_{1} \frac{\sinh \sqrt{2 \theta_{2}}}{\sqrt{2 \theta_{2}}}\right]^{-1 / 2}\right|_{\theta_{1}=0} d \theta_{2} \\
& =\frac{3}{8} \int_{0}^{\infty} \frac{\tanh ^{2} \sqrt{2 \theta_{2}}}{\sqrt{\cosh \sqrt{2 \theta_{2}}}} d \theta_{2}=1.8907,
\end{aligned}
$$




$$
\mathrm{E}\left(\tilde{U}_{1 / 2}^{2}\right)=\frac{1}{4} \mathrm{E}\left(W^{4}(1)\right)=\frac{3}{4}, \quad \mathrm{E}\left(\tilde{V}_{1 / 2}^{2}\right)=\mathrm{E}\left[\left(\int_{0}^{1} W^{2}(t) d t\right)^{2}\right]=\frac{7}{12},
$$

so that

$$
\mathrm{E}\left(\tilde{U}_{1 / 2}^{2}\right)=\frac{3}{4} \neq \mathrm{E}\left(\tilde{R}_{1 / 2}^{2}\right) \mathrm{E}\left(\tilde{V}_{1 / 2}^{2}\right)=1.8907 \times \frac{7}{12}=1.103 .
$$

The above moment property also holds for the OLSE of $\rho$ in the $\mathrm{I}(d+1)$ model:

$$
y_{j}=\rho y_{j-1}+v_{j}, \quad(1-L)^{d} v_{j}=u_{j}, \quad \text { true } \rho=1, \quad y_{k}=v_{k}=0, \quad(k \leq 0),
$$

where $d$ is a positive integer, whereas $\left\{u_{j}\right\}$ is a short-memory stationary process. Then it holds (Chan and Wei (1988)) for the OLSE $\hat{\rho}$ of $\rho$ in the above model that

$$
T(\hat{\rho}-1) \Rightarrow X_{d}=\frac{F_{d}^{2}(1) / 2}{\int_{0}^{1} F_{d}^{2}(t) d t}, \quad(d=1,2, \cdots),
$$

where $\left\{F_{d}(t)\right\}$ is the $d$-fold integrated Brownian motion defined by

$$
F_{d}(t)=\int_{0}^{t} F_{d-1}(u) d u=\frac{1}{d !} \int_{0}^{t}(t-u)^{d} d W(u), \quad(d=1,2, \cdots), \quad F_{0}(t)=W(t) .
$$

Note that $\left\{F_{d}(t)\right\}$ is, except for a constant, the same as the truncated fBm or the type II $\mathrm{fBm}\left\{B_{H}^{*}(t)\right\}$ defined in (9) when $H-1 / 2(=d)$ is a positive integer. The distribution of $X_{d}$ in (50) may be called the higher order unit root distribution.

We can now establish the following theorem concerning the mean of the higher order unit root distribution:

Theorem 6. For the OLSE of $\rho$ in the $\mathrm{I}(d+1)$ model in $(49)$, the weak convergence in (50) holds and, for $d=1,2$,

$$
\mathrm{E}\left(X_{d}\right)=\mathrm{E}\left(\frac{F_{d}^{2}(1) / 2}{\int_{0}^{1} F_{d}^{2}(t) d t}\right)=d+1
$$

Note here that

$$
\mathrm{E}\left(F_{d}^{2}(t)\right)=\frac{1}{(d !)^{2}} \int_{0}^{t}(t-u)^{2 d} d u=\frac{t^{2 d+1}}{(2 d+1)(d !)^{2}},
$$

so that Theorem 6 implies that, for $d=1,2$,

$$
\begin{aligned}
\mathrm{E}\left(X_{d}\right) & =\mathrm{E}\left(\frac{F_{d}^{2}(1) / 2}{\int_{0}^{1} F_{d}^{2}(t) d t}\right)=\frac{\mathrm{E}\left(F_{d}^{2}(1) / 2\right)}{\mathrm{E}\left(\int_{0}^{1} F_{d}^{2}(t) d t\right)}=\frac{1 / 2}{1 /(2(d+1))} \\
& =d+1
\end{aligned}
$$

Figure 7 draws the densities of $X_{d}$ for $d=1,2$ in addition to the densities of the approximate fractional unit root distributions shown in Figure 6. Note that the integration orders $d=1,2$ correspond to $H=3 / 2,5 / 2$, respectively. It is seen that the distributions are continually shifted to the right as $H$ and $d$ become large. It is really interesting to note that the means of these distributions are given by $H+1 / 2(=d+1)$. 


\section{Figure 7}

The same property is expected to hold for $d=3,4, \cdots$, but it remains to be shown because the derivation of the associated FD is much involved and is yet to be done for $d \geq 3$. It is also our conjecture that the same porperty will hold for the original fractional unit root distribution, that is,

$$
\mathrm{E}\left(R_{H}\right)=\mathrm{E}\left(\frac{B_{H}^{2}(1) / 2}{\int_{0}^{1} B_{H}^{2}(t) d t}\right)=\frac{\mathrm{E}\left(B_{H}^{2}(1) / 2\right)}{\mathrm{E}\left(\int_{0}^{1} B_{H}^{2}(t) d t\right)}=\frac{1 / 2}{1 /(2 H+1)}=H+\frac{1}{2} .
$$

\section{Concluding remarks}

We have discussed how to compute the distributions of quadratic functionals of the fBm. Its exact computation, however, turned out to be difficult. We then suggested how to approximate the distributions based on a martingale approximation, which seems to work well, but whose accuracy remains to be investigated. Our methodology was applied to compute the fractional unit root distributions. We have found that the approximate fractional as well as higher order unit root distributions have an interesting moment property. It is our conjecture that the same property will hold for original fractional unit root distributions. 
Anderson, T.W. \& D.A. Darling (1952) Asymptotic theory of certain 'goodness of fit' criteria based on stochastic processes. Annals of Mathematical Statistics 23, 193212.

Chan, N.H. \& C.Z. Wei (1988) Limiting distributions of least squares estimates of unstable autoregressive processes. Annals of Statistics 16, 367-401.

Davydov, Y. (1970) The invariance principle for stationary processes. Theory of Probability and Its Applications 15, 487-498.

Davidson, J. \& N. Hashimzade (2009) Type I and type II fractional Brownian motions: A reconsideration. Computational Statistics and Data Analysis 53, 2089-2106.

Gripenberg, G. \& I. Norros (1996) On the prediction of fractional Brownian motion. Journal of Applied Probability 33, 400-410.

Hochstadt, H. (1973) Integral Equations, New York: Wiley.

Kleptsyna, M.L., A. Le Breton \& M.C. Roubaud (2000) Parameter estimation and optimal filtering for fractional type stochastic systems. Statistical Inference for Stochastic Processes 3, 173-182.

Kleptsyna, M.L. \& A. Le Breton (2002) Statistical analysis of the fractional OrnsteinUhlenbeck type process. Statistical Inference for Stochastic Processes 5, 229-248.

Kolmogorov, A.N. (1940) Wienersche Spiralen und einige andere interessante Kurven im Hilbertschen Raum. Dokl. Acad. Nauk. SSSR 26, 115-118.

Liptser, R.S. \& A.N. Shiryaev (1977) Statistics of Random Processes I: General Theory, New York: Springer-Verlag.

Loève, M. (1978) Probability Theory II, Fourth Edition, New York: Springer-Verlag.

Mandelbrot, B.B. \& J.W. Van Ness (1968) Fractional Brownian motions, fractional noises and applications. SIAM Review 10, 422-437.

Marinucci, D. \& P.M. Robinson (1999) Alternative forms of fractional Brownian motion. Journal of Statistical Inference and Planning 80, 111-122.

Nabeya, S. \& K. Tanaka (1988) Asymptotic theory of a test for the constancy of regression coefficients against the random walk alternative. Annals of Statistics 16, 218-235.

Nabeya, S. \& K. Tanaka (1990) A general approach to the limiting distribution for estimators in time series regression with nonstable autoregressive errors. Econometrica $58,145-163$.

Norros, I., E. Valkeila \& J. Virtamo (1999) An elementary approach to a Girsanov formula and other analytical results on fractional Brownian motions. Bernoulli 5, 571-587. 
Sowell, F. (1990) The fractional unit root distribution. Econometrica 58, 495-505.

Tanaka, K. (1996) Time Series Analysis: Nonstationary and Noninvertible Distribution Theory, New York: Wiley.

Watson, G.N. (1958) A Treatise on the Theory of Bessel Functions, Second Edition, London: Cambridge University Press.

Watson, G.S. (1961) Goodness-of-fit tests on a circle. Biometrika 48, 109-114. 


\section{Appendix}

Proof of Theorem 3. We first work with the kernel in (39):

$$
K_{A}(s, t)=\frac{1-2 \alpha}{4 \alpha+1}\left(1-(\max (s, t))^{4 \alpha+1}\right) s^{-\alpha} t^{-\alpha} .
$$

The integral equation (12) with the kernel $K_{A}(s, t)$ in (A.1) leads us to derive the following differential equation and two boundary conditions.

$$
\begin{aligned}
& h^{\prime \prime}(t)+\left(\lambda(1-2 \alpha) t^{2 \alpha}-\frac{2 \alpha(2 \alpha+1)}{t^{2}}\right) h(t)=0, \\
& h(1)=0, \quad \lim _{t \rightarrow 0}\left(2 \alpha t^{-2 \alpha-1} h(t)+t^{-2 \alpha} h^{\prime}(t)\right)=0,
\end{aligned}
$$

where we have put $h(t)=f(t) / t^{\alpha}$. Using the fact that

$$
\left(2 \alpha t^{-2 \alpha-1} h(t)+t^{-2 \alpha} h^{\prime}(t)\right)^{\prime}=-\lambda(1-2 \alpha) h(t),
$$

it can be shown that the differential equation (A.2) with two boundary conditions is equivalent to the integral equation. The general solution to (A.2) is given (Watson (1958)) by

$$
h(t)=t^{1 / 2}\left(c_{1} J_{\nu}\left(\eta t^{\alpha+1}\right)+c_{2} J_{-\nu}\left(\eta t^{\alpha+1}\right)\right)
$$

where $\eta=\sqrt{\lambda(1-2 \alpha)} /(\alpha+1), \nu=(2 \alpha+1 / 2) /(\alpha+1)$, whereas $c_{1}$ and $c_{2}$ are arbitrary constants. From the two boundary conditions we have the homogeneous equation on $\boldsymbol{c}=\left(c_{1}, c_{2}\right)^{\prime}$ :

$$
M_{A}(\lambda) \boldsymbol{c}=\left(\begin{array}{cc}
J_{\nu}(\eta) & J_{-\nu}(\eta) \\
1 & 0
\end{array}\right) \boldsymbol{c}=\mathbf{0} .
$$

It follows from $\left|M_{A}(\lambda)\right|$ and using the fact (Watson (1958)) that

$$
J_{-\nu}(z)=\frac{1}{\Gamma(1-\nu)(z / 2)^{\nu}} \prod_{n=1}^{\infty}\left(1-\frac{z^{2}}{a_{n}^{2}}\right),
$$

where $0<a_{1}<a_{2}<\cdots$ are positive zeros of $J_{-\nu}(z)$, we obtain, as the FD of $K_{A}$,

$$
D_{H}(\lambda)=\left(\frac{\eta}{2}\right)^{\nu} \Gamma(1-\nu) J_{-\nu}(\eta)
$$

We next consider the kernel in (40):

$$
K_{B}(s, t)=(s t)^{2 \alpha}(\min (s, t))^{1-2 \alpha},
$$

which gives us the following differential equation and boundary conditions:

$$
\begin{aligned}
& f^{\prime \prime}(t)-\frac{2 \alpha}{t} f^{\prime}(t)+\left(\lambda(1-2 \alpha) t^{2 \alpha}+\frac{2 \alpha}{t^{2}}\right) f(t)=0, \\
& \lim _{t \rightarrow 0} t^{1-2 \alpha} f^{\prime}(t)=0, \quad f^{\prime}(1)=2 \alpha f(1) .
\end{aligned}
$$


The general solution to (A.4) is given by

$$
f(t)=t^{\alpha+1 / 2}\left(c_{1} J_{\nu-1}\left(\eta t^{\alpha+1}\right)+c_{2} J_{1-\nu}\left(\eta t^{\alpha+1}\right)\right),
$$

where $\eta$ and $\nu$ are the same as before, whereas $c_{1}$ and $c_{2}$ are arbitrary constants. We then have, from the two boundary conditions, the homogeneous equation on $\boldsymbol{c}=\left(c_{1}, c_{2}\right)^{\prime}$ :

$$
M_{B}(\lambda) \boldsymbol{c}=\left(\begin{array}{cc}
1 & 0 \\
a & \frac{1-2 \alpha}{\eta(\alpha+1)} J_{1-\nu}(\eta)-J_{2-\nu}(\eta)
\end{array}\right) \boldsymbol{c}
$$

where $a$ is some constant. Since it holds (Watson (1958)) that

$$
J_{\nu}(z)=\frac{2(\nu+1)}{z} J_{\nu+1}(z)-J_{\nu+2}(z)
$$

it is seen that

$$
J_{-\nu}(\eta)=\frac{1-2 \alpha}{\eta(\alpha+1)} J_{1-\nu}(\eta)-J_{2-\nu}(\eta)
$$

Thus, computing $\left|M_{B}(\lambda)\right|$, we obtain, as the FD of $K_{B}$, the same FD as that of $K_{A}$. In terms of numerical computations, it may be convenient to compute $J_{-\nu}(\eta)$ using the expression on the right side of (A.5). This completes the proof of Theorem 3.

Proof of Theorem 4. Putting $h(t)=f(t) / t^{\alpha}$, the integral equation (12) with the kernel $K_{H}(s, t ; x)$ in $(45)$ takes the form:

$$
\begin{aligned}
t^{2 \alpha} h(t)= & \lambda\left[\frac{x}{4 \alpha+1}\left\{\int_{0}^{1} h(s) d s-t^{4 \alpha+1} \int_{0}^{t} h(s) d s-\int_{t}^{1} s^{4 \alpha+1} h(s) d s\right\}\right. \\
& \left.-\frac{1}{2} \int_{0}^{1} h(s) d s\right] .
\end{aligned}
$$

The above integral equation is ensured to be equivalent to

$$
\begin{aligned}
& h^{\prime \prime}(t)+\left(\lambda x t^{2 \alpha}-\frac{2 \alpha(2 \alpha+1)}{t^{2}}\right) h(t)=0, \\
& 2(\alpha-x) h(1)+h^{\prime}(1)=0, \quad \lim _{t \rightarrow 0}\left(2 \alpha t^{-2 \alpha-1} h(t)+t^{-2 \alpha} h^{\prime}(t)\right)=\lim _{t \rightarrow 0} G(t)=0,
\end{aligned}
$$

In fact, the equivalence may be proved by noting that

$$
\left(2 \alpha t^{-2 \alpha-1} h(t)+t^{-2 \alpha} h^{\prime}(t)\right)^{\prime}=G^{\prime}(t)=g(t)=-\lambda x h(t) .
$$

Then the right side of (A.6) denoted as RH becomes

$$
\begin{aligned}
\mathrm{RH} & =\frac{1}{4 \alpha+1}\left\{-\int_{0}^{1} g(s) d s+t^{4 \alpha+1} \int_{0}^{t} g(s) d s+\int_{t}^{1} s^{4 \alpha+1} g(s) d s\right\}+\frac{1}{2 x} \int_{0}^{1} g(s) d s \\
& =-\int_{t}^{1} s^{4 \alpha} G(s) d s+\frac{1}{2 x} G(1) \\
& =-\int_{0}^{1} s^{4 \alpha}\left(2 \alpha s^{-2 \alpha-1} h(s)+s^{-2 \alpha} h^{\prime}(s)\right) d s+h(1) \\
& =t^{2 \alpha} h(t)
\end{aligned}
$$


where we have used the two boundary conditions $G(1)=2 x h(1)$ and $G(0)=0$.

The general solution to (A.7) is given by

$$
h(t)=t^{1 / 2}\left(c_{1} J_{\nu}\left(\kappa t^{\alpha+1}\right)+c_{2} J_{-\nu}\left(\kappa t^{\alpha+1}\right)\right),
$$

where $\nu=(2 \alpha+1 / 2) /(\alpha+1), \kappa=\sqrt{\lambda x} /(\alpha+1)$, whereas $c_{1}$ and $c_{2}$ are arbitrary constants. Then the two boundary conditions give us the homogeneous equation on $\boldsymbol{c}=\left(c_{1}, c_{2}\right)^{\prime}$ :

$$
M(\lambda) \boldsymbol{c}=\left(\begin{array}{cc}
b & -\left(2 x J_{-\nu}(\kappa)+\sqrt{\lambda x} J_{1-\nu}(\kappa)\right) \\
1 & 0
\end{array}\right)=\mathbf{0},
$$

where $b$ is some constant. Let the positive zeros of $J_{-\nu}(\kappa)$ and $J_{1-\nu}(\kappa)$ be $0<a_{1}<a_{2}<\cdots$ and $0<b_{1}<b_{2}<\cdots$, respectively. Then it follows from Watson (1958) that

$$
J_{-\nu}(\kappa)=\frac{(\kappa / 2)^{-\nu}}{\Gamma(1-\nu)} \prod_{n=1}^{\infty}\left(1-\frac{\kappa^{2}}{a_{n}^{2}}\right), \quad J_{1-\nu}(\kappa)=\frac{(\kappa / 2)^{1-\nu}}{\Gamma(2-\nu)} \prod_{n=1}^{\infty}\left(1-\frac{\kappa^{2}}{b_{n}^{2}}\right) .
$$

Thus we have

$$
\begin{aligned}
|M(\lambda)| & =2 x J_{-\nu}(\kappa)+\sqrt{\lambda x} J_{1-\nu}(\kappa) \\
& =\frac{(\kappa / 2)^{-\nu}}{\Gamma(1-\nu)}\left[2 x \prod_{n=1}^{\infty}\left(1-\frac{\kappa^{2}}{a_{n}^{2}}\right)+\frac{\kappa / 2}{1-\nu} \sqrt{\lambda x} \prod_{n=1}^{\infty}\left(1-\frac{\kappa^{2}}{b_{n}^{2}}\right)\right] \\
& =\frac{2 x(\kappa / 2)^{-\nu}}{\Gamma(1-\nu)}\left[\prod_{n=1}^{\infty}\left(1-\frac{\kappa^{2}}{a_{n}^{2}}\right)+\frac{\lambda}{2(1-2 \alpha)} \prod_{n=1}^{\infty}\left(1-\frac{\kappa^{2}}{b_{n}^{2}}\right)\right],
\end{aligned}
$$

and we obtain, as the FD of $K_{H}(s, t ; x)$,

$$
\begin{aligned}
D_{H}(\lambda ; x) & =\prod_{n=1}^{\infty}\left(1-\frac{\kappa^{2}}{a_{n}^{2}}\right)+\frac{\lambda}{2(1-2 \alpha)} \prod_{n=1}^{\infty}\left(1-\frac{\kappa^{2}}{b_{n}^{2}}\right) \\
& =\frac{\Gamma(1-\nu)}{2 x(\kappa / 2)^{-\nu}}\left[2 x J_{-\nu}(\kappa)+\sqrt{\lambda x} J_{1-\nu}(\kappa)\right] \\
& =\left(\frac{\kappa}{2}\right)^{\nu} \Gamma(1-\nu)\left(J_{-\nu}(\kappa)+\frac{\lambda}{2} \frac{J_{1-\nu}(\kappa)}{\sqrt{\lambda x}}\right),
\end{aligned}
$$

which establishes Theorem 4.

Proof of Theorem 5. Since we have

$$
m\left(\theta_{1},-\theta_{2}\right)=\left[\left(\frac{\xi}{2}\right)^{\nu} \Gamma(1-\nu)\left(J_{-\nu}(\xi)-\frac{\theta_{1}}{(\alpha+1) \xi} J_{1-\nu}(\xi)\right)\right]^{-1 / 2}
$$

where $\xi=\sqrt{-2 \theta_{2}} /(\alpha+1)$, we obtain

$$
\begin{aligned}
\left.\frac{\partial m\left(\theta_{1},-\theta_{2}\right)}{\partial \theta_{1}}\right|_{\theta_{1}=0}= & \frac{1}{2}\left(\frac{\xi}{2}\right)^{-\nu / 2} \frac{(\Gamma(1-\nu))^{-1 / 2}}{(\alpha+1) \xi} J_{1-\nu}(\xi)\left(J_{-\nu}(\xi)\right)^{-3 / 2} \\
= & {\left[\left(\frac{\eta \sqrt{\theta_{2}}}{2}\right)^{\nu} \Gamma(1-\nu) J_{-\nu}\left(\eta \sqrt{\theta_{2}}\right)\right]^{-3 / 2} } \\
& \times\left\{-\frac{\eta^{2}}{2^{\nu+1}} \Gamma(1-\nu)\left(\eta \sqrt{\theta_{2}}\right)^{\nu-1} J_{1-\nu}\left(\eta \sqrt{\theta_{2}}\right)\right\} \times \frac{\alpha+1}{2},
\end{aligned}
$$


where we have put $\eta=\sqrt{-2} /(\alpha+1)$. Using the following formula (Watson (1958))

$$
\frac{d\left\{z^{\nu} J_{-\nu}(z)\right\}}{d z}=-z J_{1-\nu}(z)
$$

it can be shown that

$$
\frac{d}{d \theta_{2}}\left(\left(\eta \sqrt{\theta_{2}}\right)^{\nu} J_{-\nu}\left(\eta \sqrt{\theta_{2}}\right)\right)=-\frac{1}{2} \eta^{\nu+1} \theta_{2}^{(\nu-1) / 2} J_{1-\nu}\left(\eta \sqrt{\theta_{2}}\right) .
$$

Considering the following expansion and the change of variables

$$
\begin{aligned}
u & =\left(\frac{\eta \sqrt{\theta_{2}}}{2}\right)^{\nu} \Gamma(1-\nu) J_{-\nu}\left(\eta \sqrt{\theta_{2}}\right)=\prod_{n=1}^{\infty}\left(1-\frac{\eta^{2} \theta_{2}}{a_{n}^{2}}\right) \\
& =\prod_{n=1}^{\infty}\left(1+\frac{2 \theta_{2}}{(\alpha+1)^{2} a_{n}^{2}}\right),
\end{aligned}
$$

we have $1<u<\infty$ for $0<\theta_{2}<\infty$ and

$$
d u=-\frac{\eta^{2}}{2^{\nu+1}} \Gamma(1-\nu)\left(\eta \sqrt{\theta_{2}}\right)^{\nu-1} J_{1-\nu}\left(\eta \sqrt{\theta_{2}}\right) d \theta_{2} .
$$

Thus we finally arrive at

$$
\begin{aligned}
\mathrm{E}\left(\tilde{R}_{H}\right)= & \left.\int_{0}^{\infty} \frac{\partial m\left(\theta_{1},-\theta_{2}\right)}{\partial \theta_{1}}\right|_{\theta_{1}=0} d \theta_{2} \\
= & \int_{0}^{\infty}\left[\left(\frac{\eta \sqrt{\theta_{2}}}{2}\right)^{\nu} \Gamma(1-\nu) J_{-\nu}\left(\eta \sqrt{\theta_{2}}\right)\right]^{-3 / 2} \\
& \times\left\{-\frac{\eta^{2}}{2^{\nu+1}} \Gamma(1-\nu)\left(\eta \sqrt{\theta_{2}}\right)^{\nu-1} J_{1-\nu}\left(\eta \sqrt{\theta_{2}}\right)\right\} \times \frac{\alpha+1}{2} d \theta_{2} \\
= & \frac{\alpha+1}{2} \int_{1}^{\infty} u^{-3 / 2} d u \\
= & \alpha+1=H+\frac{1}{2}
\end{aligned}
$$

which proves the theorem.

Proof of Theorem 6. Let us define

$$
m_{d}\left(\theta_{1}, \theta_{2}\right)=\mathrm{E}\left[\exp \left\{\frac{\theta_{1}}{2} F_{d}^{2}(1)+\theta_{2} \int_{0}^{1} F_{d}^{2}(t) d t\right\}\right] \text {. }
$$

Then it follows from Tanaka (1996, p.275) that

$$
\begin{aligned}
m_{1}\left(\theta_{1},-\theta_{2}\right)= & {\left[\frac{1}{2}(1+\cos \mu \cosh \mu)+\frac{\theta_{1}}{4 \theta_{2}}(\mu \cosh \mu \sin \mu-\mu \cos \mu \sinh \mu)\right]^{-1 / 2}, } \\
m_{2}\left(\theta_{1},-\theta_{2}\right)= & {\left[\frac { 1 } { 1 8 } \left\{4\left(1+\cos \nu+\cos \nu \omega+\cos \nu \omega^{2}\right)+2 \cos \nu \cos \nu \omega \cos \nu \omega^{2}\right.\right.} \\
& +\frac{\theta_{1}}{\theta_{2}}\left(\frac{1}{2}\left(\nu \sin 2 \nu+\nu \omega \sin 2 \nu \omega+\nu \omega^{2} \sin 2 \nu \omega^{2}\right)\right. \\
& \left.\left.\left.+2\left(\nu \sin \nu+\nu \omega \sin \nu \omega+\nu \omega^{2} \sin \nu \omega^{2}\right)\right)\right\}\right]^{-1 / 2}
\end{aligned}
$$


where

$$
\mu=\left(-2 \theta_{2}\right)^{1 / 4}, \quad \nu=\left(-2 \theta_{2}\right)^{1 / 6}, \quad \omega=\frac{1+\sqrt{3} i}{2} .
$$

Thus we have $\partial m_{d}\left(\theta_{1},-\theta_{2}\right) /\left.\partial \theta_{1}\right|_{\theta_{1}=0}=A_{d}\left(\theta_{2}\right)\left(B_{d}\left(\theta_{2}\right)\right)^{-3 / 2}$, where

$$
\begin{aligned}
A_{1}\left(\theta_{2}\right)= & -\frac{1}{8 \theta_{2}}(\mu \cosh \mu \sin \mu-\mu \cos \mu \sinh \mu), \\
B_{1}\left(\theta_{2}\right)= & \frac{1}{2}(1+\cos \mu \cosh \mu), \\
A_{2}\left(\theta_{2}\right)= & -\frac{1}{36 \theta_{2}}\left(\frac{1}{2}\left(\nu \sin 2 \nu+\nu \omega \sin 2 \nu \omega+\nu \omega^{2} \sin 2 \nu \omega^{2}\right)\right. \\
& \left.+2\left(\nu \sin \nu+\nu \omega \sin \nu \omega+\nu \omega^{2} \sin \nu \omega^{2}\right)\right), \\
B_{2}\left(\theta_{2}\right)= & \frac{1}{9}\left\{2\left(1+\cos \nu+\cos \nu \omega+\cos \nu \omega^{2}\right)+\cos \nu \cos \nu \omega \cos \nu \omega^{2}\right\}
\end{aligned}
$$

Putting $u=B_{1}\left(\theta_{2}\right)$ and $v=B_{2}\left(\theta_{2}\right)$, it can be checked after some algebra that

$$
d u=A_{1}\left(\theta_{2}\right) d \theta_{2}, \quad d v=\frac{3}{2} A_{2}\left(\theta_{2}\right) d \theta_{2} .
$$

We can now compute

$$
\begin{aligned}
\mathrm{E}\left(X_{d}\right) & =\mathrm{E}\left(\frac{F_{d}^{2}(1) / 2}{\int_{0}^{1} F_{d}^{2}(t) d t}\right)=\left.\int_{0}^{\infty} \frac{\partial m_{d}\left(\theta_{1},-\theta_{2}\right)}{\partial \theta_{1}}\right|_{\theta_{1}=0} d \theta_{2} \\
& =\int_{0}^{\infty} A_{d}\left(\theta_{2}\right)\left(B_{d}\left(\theta_{2}\right)\right)^{-3 / 2} d \theta_{2}=\frac{d+1}{2} \int_{1}^{\infty} u^{-3 / 2} d u \\
& =d+1, \quad(d=1,2),
\end{aligned}
$$

which completes the proof of Theorem 6 . 
Table 1. Comparison of variances of $S_{H}$ and $\tilde{S}_{H}$

\begin{tabular}{cccccccc}
$H$ & 0.5 & 0.6 & 0.7 & 0.8 & 0.9 & 0.95 & 1.0 \\
\hline $\mathrm{V}\left(S_{H}\right)$ & $1 / 3$ & 0.30652 & 0.28194 & 0.25975 & 0.23990 & 0.23081 & $2 / 9$ \\
$\mathrm{~V}\left(\tilde{S}_{H}\right)$ & $1 / 3$ & 0.30303 & 0.27778 & 0.25641 & 0.23810 & 0.22989 & $2 / 9$ \\
\hline
\end{tabular}

Table 2. Percent points of $\tilde{S}_{H}$

\begin{tabular}{|c|c|c|c|c|c|c|c|}
\hline & \multicolumn{3}{|c|}{ Probability } & $\begin{array}{ll}\mathrm{f} \\
\mathrm{a}\end{array}$ & smaller & \multicolumn{2}{|l|}{ value } \\
\hline & 0.01 & 0.05 & 0.1 & 0.5 & 0.9 & 0.95 & 0.99 \\
\hline$H=0.5$ & 0.0345 & 0.0565 & 0.0765 & 0.2905 & 1.1958 & 1.6557 & 2.7875 \\
\hline$H=0.6$ & 0.0246 & 0.04160 & 0.0577 & 0.2502 & 1.1181 & 1.5582 & 2.6407 \\
\hline$H=0.7$ & 0.0169 & 0.0296 & 0.0423 & 0.2175 & 1.0521 & 1.4744 & 2.5131 \\
\hline$H=0.8$ & 0.0106 & 0.0196 & 0.0292 & 0.1909 & 0.9952 & 1.4015 & 2.4008 \\
\hline$H=0.9$ & 0.0053 & 0.0108 & 0.0174 & 0.1694 & 0.9456 & 1.3374 & 2.3011 \\
\hline$H=0.95$ & 0.0029 & 0.0064 & 0.0115 & 0.1601 & 0.9231 & 1.3081 & 2.2551 \\
\hline$H=1.0$ & 0.00047 & 0.0118 & 0.0474 & 1.3648 & 8.1166 & 11.5244 & 19.9047 \\
\hline
\end{tabular}




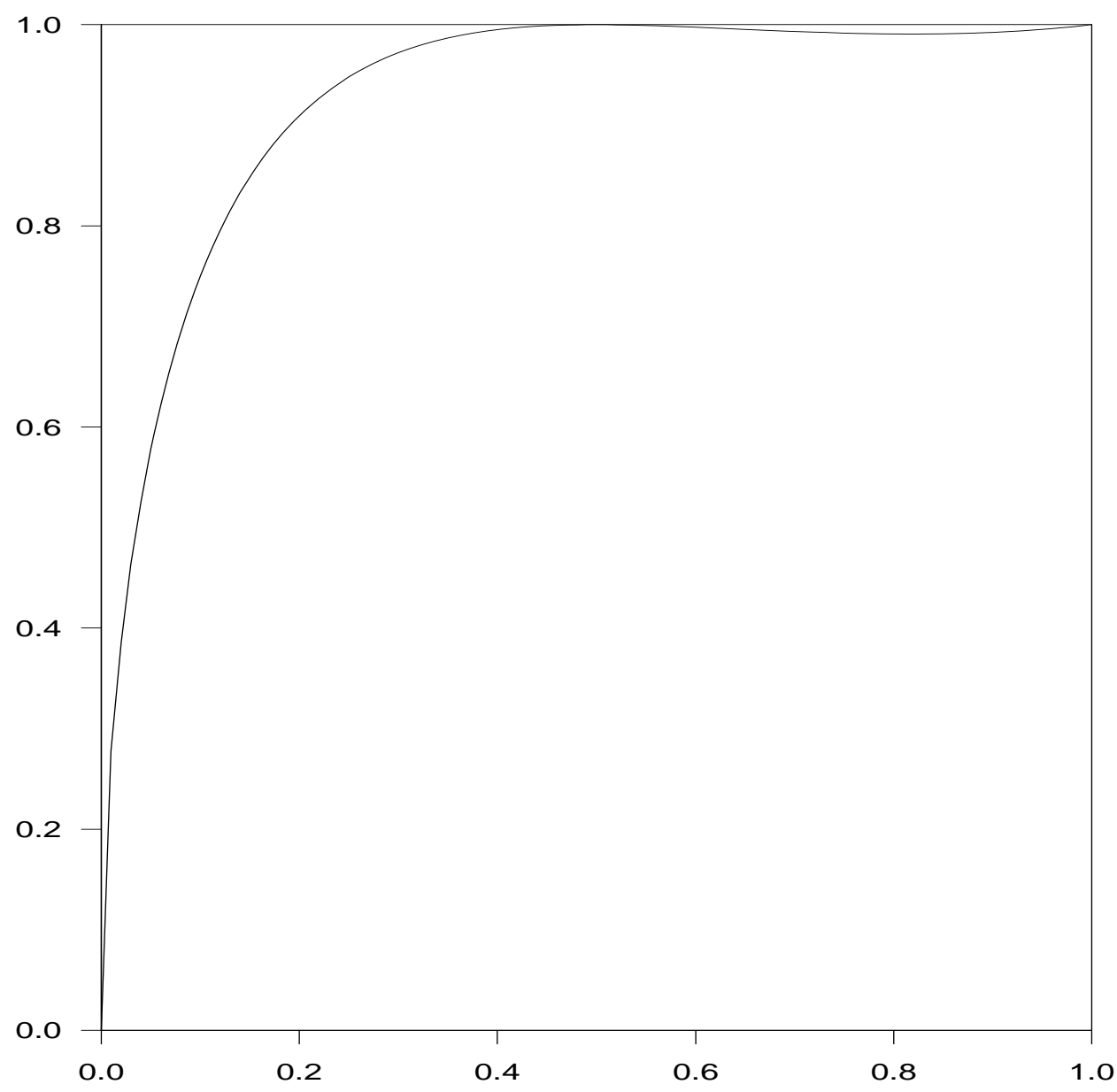

Figure 1. Correlation of $B_{H}(t)$ and $M_{H}(t)$ as a function of $H$ 


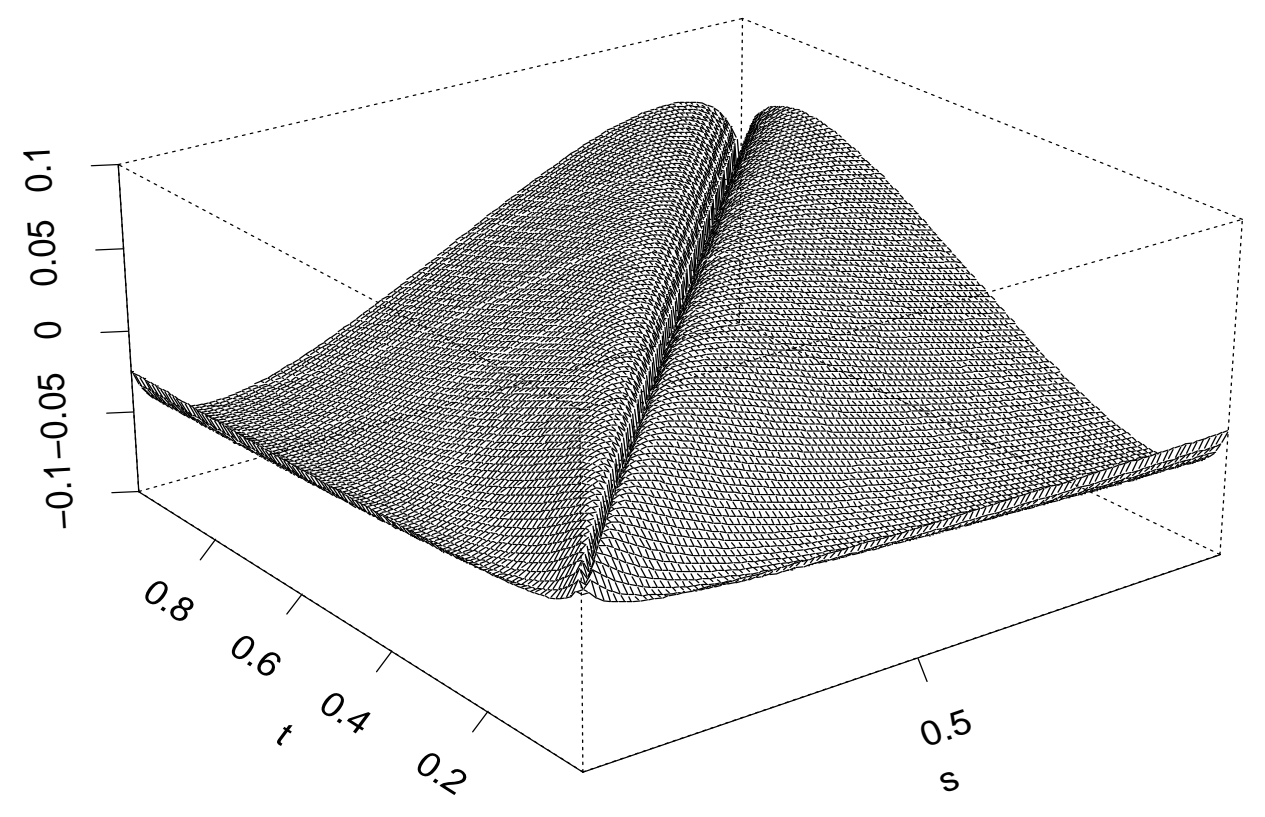

Figure 2. $D_{H}(s, t)$ for $H=0.3$ 


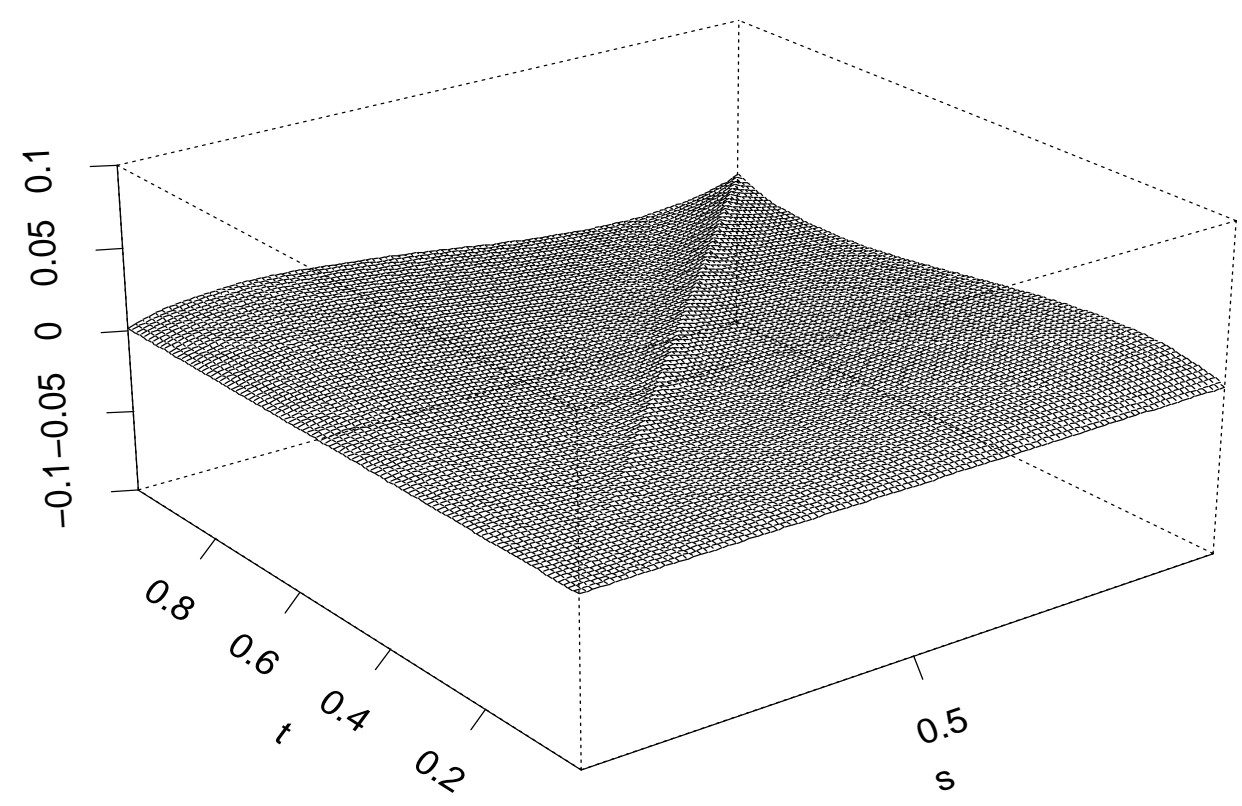

Figure 3. $D_{H}(s, t)$ for $H=0.8$ 


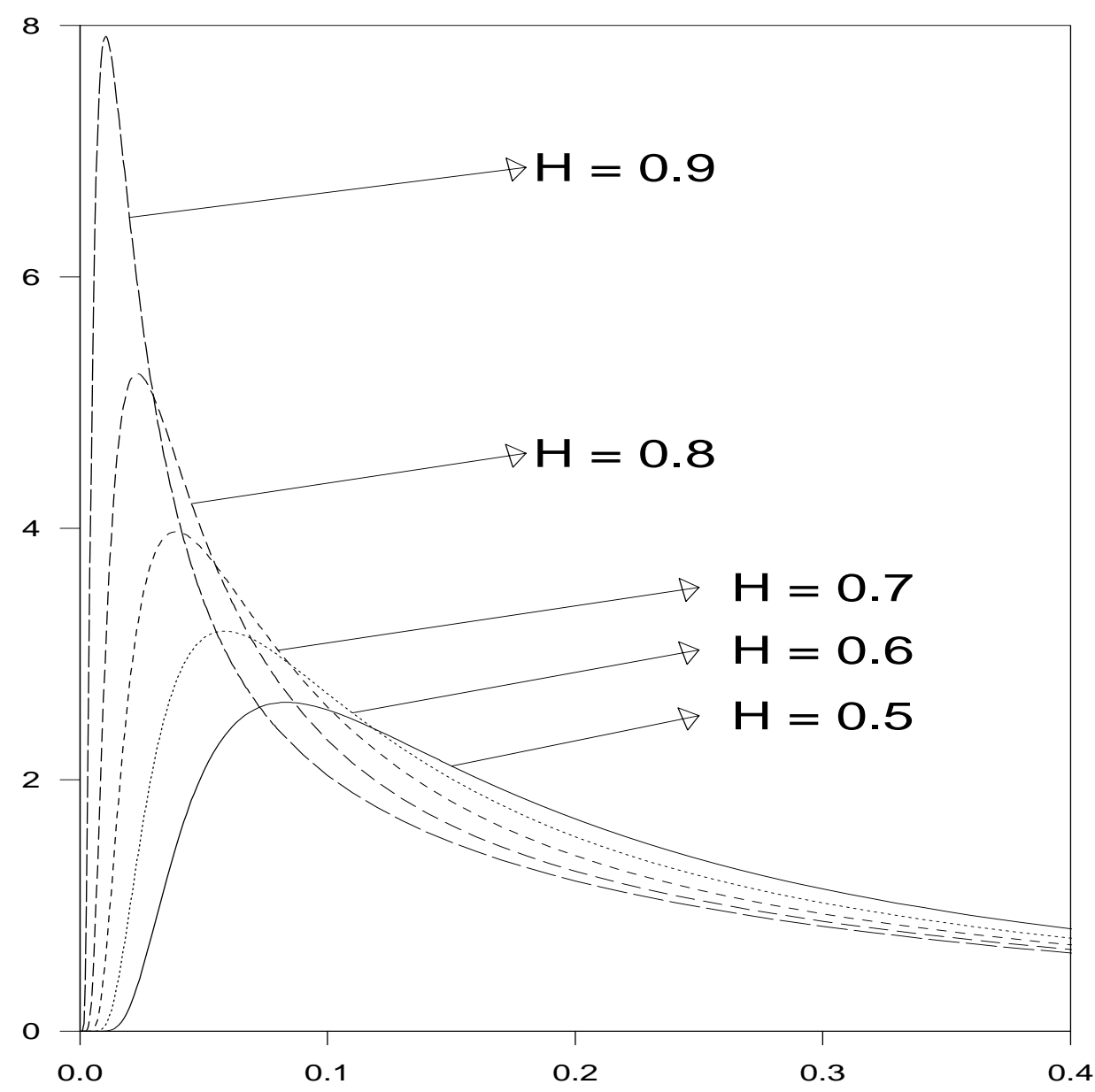

Figure 4. Approximate densities of $\int_{0}^{1} B_{H}^{2}(t) d t$ for various values of $H(\geq 1 / 2)$ 


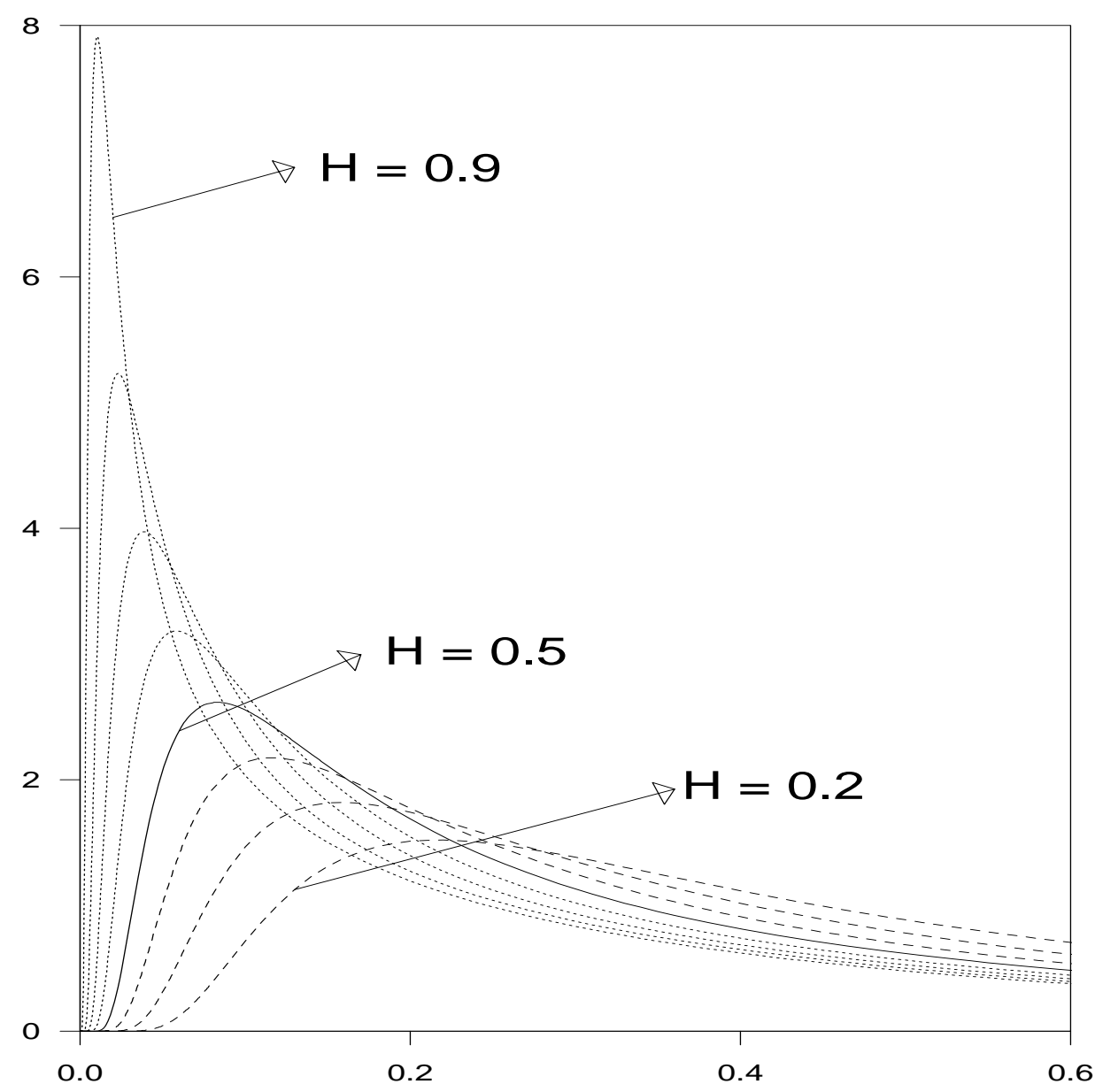

Figure 5. Densities of $\tilde{S}_{H}$ for $0<H<1$ 


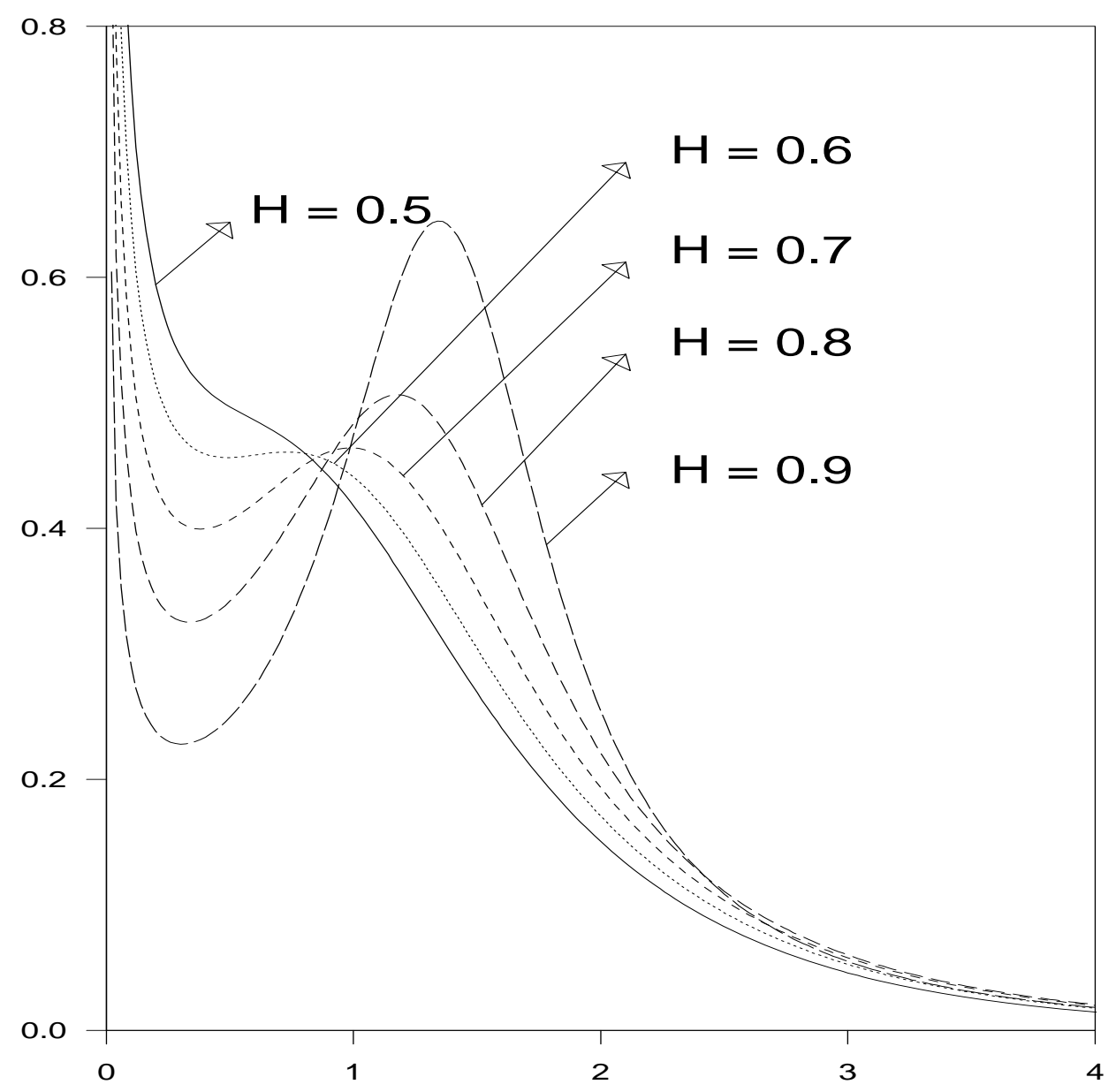

Figure 6. Approximate densities of the fractional unit root distributions 


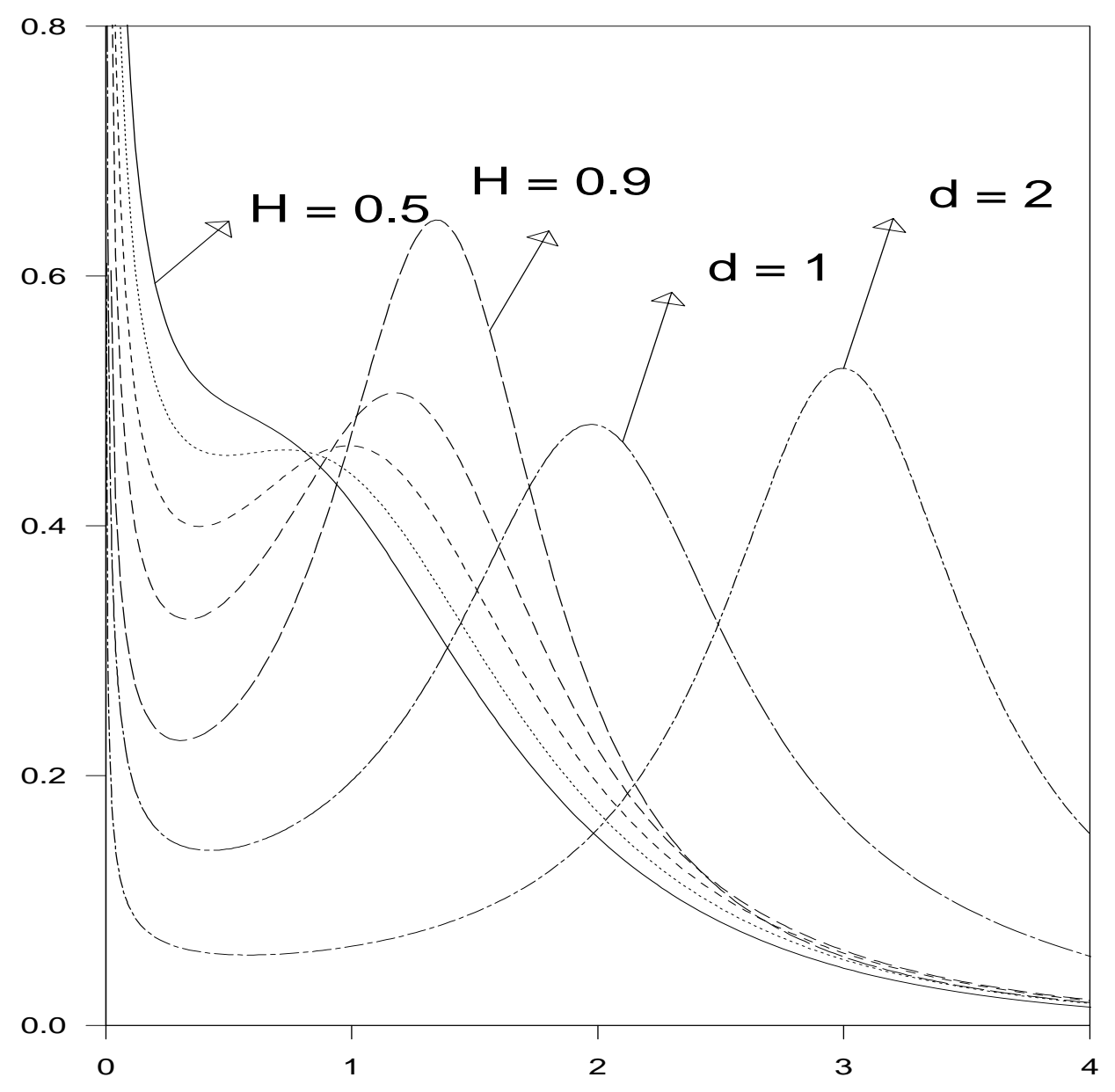

Figure 7. Fractional and higher order unit root distributions 